\title{
Spectrum sharing on interference channels with a cognitive relay
}

\author{
Qiang Li ${ }^{1}$, Ashish Pandharipande 2 , See Ho Ting ${ }^{3}$ and Xiaohu Ge ${ }^{\text {* }}$
}

\begin{abstract}
In this paper, an interference channel with a cognitive relay (IFC-CR) is considered to achieve spectrum sharing between a licensed primary user and an unlicensed secondary user. The CR assists both users in relaying their messages to the respective receivers, under the constraint that the performance of the legacy primary user is not degraded. Without requiring any non-causal knowledge, the CR uses a successive interference cancellation to first decode the primary and secondary messages after a transmission phase. A power allocation is then performed to forward a linear weighted combination of the processed signals in the relaying phase. Closed-form expressions of the end-to-end outage probability are derived for both primary and secondary users under the proposed approach. Furthermore, by exploiting the decoded primary and secondary messages in the first phase, we propose the use of dirty paper coding (DPC) at CR to pre-cancel the interference seen at the secondary (or primary) receiver in the second phase, which results in a performance upper bound for the secondary (or primary) user without affecting the other user. Simulation results demonstrate that with a joint consideration of the power control at the secondary transmitter and the power allocation at CR, performance gains can be achieved for both primary and secondary users.
\end{abstract}

Keywords: Cognitive spectrum sharing; Interference channel with a cognitive relay; Successive interference cancellation; Dirty paper coding

\section{Introduction}

\subsection{Background and related work}

Consider a spectrum sharing system, as shown in Fig. 1, where two interfering users co-exist with a cognitive relay in the same frequency band. For both users to operate properly, the cognitive relay $[1,2]$ assists in forwarding the messages from both transmitters to their respective receivers [3-6] and at the same time coordinates the mutual interference. This constitutes an interference channel with a cognitive relay (IFC-CR), which has been intensively studied from an information-theoretic perspective [7-15].

A two-user symmetric Gaussian IFC-CR was first introduced in [7], where the CR was assumed to be full duplex and adopt a decode-and-forward (DF) processing. Through rate splitting [16] at both sources and joint decoding at each destination, an achievable rate region was obtained. Then, this achievable rate was improved

*Correspondence: xhge@hust.edu.cn

1 school of Electronic Information and Communications, Huazhong University of Science and Technology, Wuhan, China

Full list of author information is available at the end of the article in [8] by performing sophisticated coding strategies that require non-causal information of both transmitters at the $\mathrm{CR}$ prior to information transmission. By combining the Han-Kobayashi coding scheme [16] for interference channels with dirty paper coding (DPC) [17], a generalization of the achievable rate region obtained in [8] was derived in [9]. In [10], an outer bound for the capacity of a general IFC-CR was first derived. New inner and outer bounds for the capacity of IFC-CR were derived later in [11-15], under various conditions.

A Gaussian interference channel with an out-of-band relay was investigated in $[18,19]$ where the relay was assumed to operate over orthogonal bands to the underlying interference channel. In [18], the entire system was characterized by two parallel channels, namely a Gaussian interference channel and a Gaussian relay channel. To characterize the capacity, relay operations were optimized with separable or nonseparable encoding between the interference channel and the out-of-band relay channel. In [19], the impact of the out-of-band relay channel and the corresponding signal interactions on the capacity were investigated under general channel conditions.

\section{量 Springer}




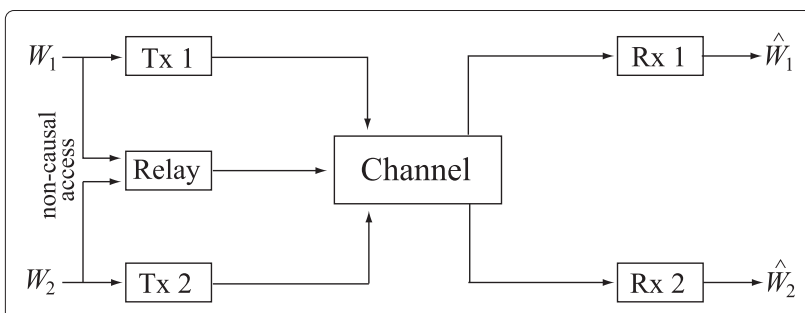

Fig. 1 An interference channel with a cognitive relay (IFC-CR) where the $C R$ has perfect non-causal knowledge of the messages originated from both transmitters

In the above works, the two interfering users are assumed to be part of the peer users in the same radio system. A question that arises is as follows: what if the two interfering users belong to different radio systems that are of different priorities. In view of the mutual interference between the two users and the inherent cognition and cooperation ability equipped at the $\mathrm{CR}$, it is natural to evaluate IFC-CR under a cognitive spectrum sharing setup between, e.g., a licensed primary user and an unlicensed secondary user [20-23]. Under such circumstances, we assume that the CR belongs to the secondary system or a third-party agent. Then, instead of characterizing the capacity or sum rate of the entire system as in [7-15], it is more pragmatic to enhance the performance of the secondary user under the constraint that no harm is caused to the legacy primary user [24-26].

A spectrum sharing protocol was proposed on the interference channel in [26]. With the assumption that the secondary transmitter has non-causal knowledge of the codewords originated at the primary transmitter, achievable rates of the secondary user were characterized under the constraint that no rate degradation was created for the primary system. As a variant of [26], a spectrum sharing protocol was proposed between a primary and a secondary user on an IFC-CR in [27]. With the assumption that non-causal knowledge of the primary codewords is available at both the secondary transmitter and the CR, an enhanced throughput was achieved for the secondary user without degrading the throughput of the primary user. In [28], a spectrum sharing protocol was proposed on an IFC-CR where the CR helps both the primary and secondary transmissions. A DF relay protocol was considered where only when both primary and secondary messages are successfully decoded at CR, they are forwarded in the second phase with a certain power allocation. Conditioned on the decoding results at CR, the received SNR at each receiver was analyzed, through which an upper bound of the outage probability was derived. In [29], an opportunistic adaptive relaying protocol is proposed on IFC-CR, where CR is able to determine when to cooperate with the primary user, when to cooperate with the secondary user, and when to cooperate with both users simultaneously. An upper bound of the secondary outage probability was derived under a primary outage probability threshold. In [30], an amplify-and-forward (AF) relay protocol was performed at $\mathrm{CR}$ to help relay the signals of both primary and secondary users over independent Nakagami- $m$ fading channels. Assuming there are no cross links between primary and secondary users, endto-end outage probabilities of the primary and secondary users were obtained. Simulation results demonstrated a performance gain for both primary and secondary users.

\subsection{Our contributions}

Motivated by the above works, we propose a causal cognitive spectrum sharing protocol on a fading IFC-CR [31]. As depicted in Fig. 2, both primary transmitter (PT) and secondary transmitter (ST) transmit simultaneously in the first phase. Without requiring non-causal knowledge of the messages from PT or ST, CR attempts to decode both messages using successive interference cancellation (SIC) [32]. To compensate the interference seen at the primary receiver (PR) that is caused by cross talk, we consider a power allocation scheme at CR. To be specific, a hybrid AF-DF relay protocol [33] is considered such that when at least one of the two messages is successfully decoded, a fraction $\alpha, 0<\alpha<1$, of the transmit power of CR is used to forward the primary signal, with the remaining power to forward the secondary signal, in the second phase. If however, neither of the messages is decoded, the CR simply stays silent and both PT and ST perform a retransmission simultaneously in the second phase.

At the end of the second phase, by exploiting the received signals in two phases, maximal-ratio combining (MRC) is employed at PR to decode the desired message. Without incurring additional overhead to the legacy

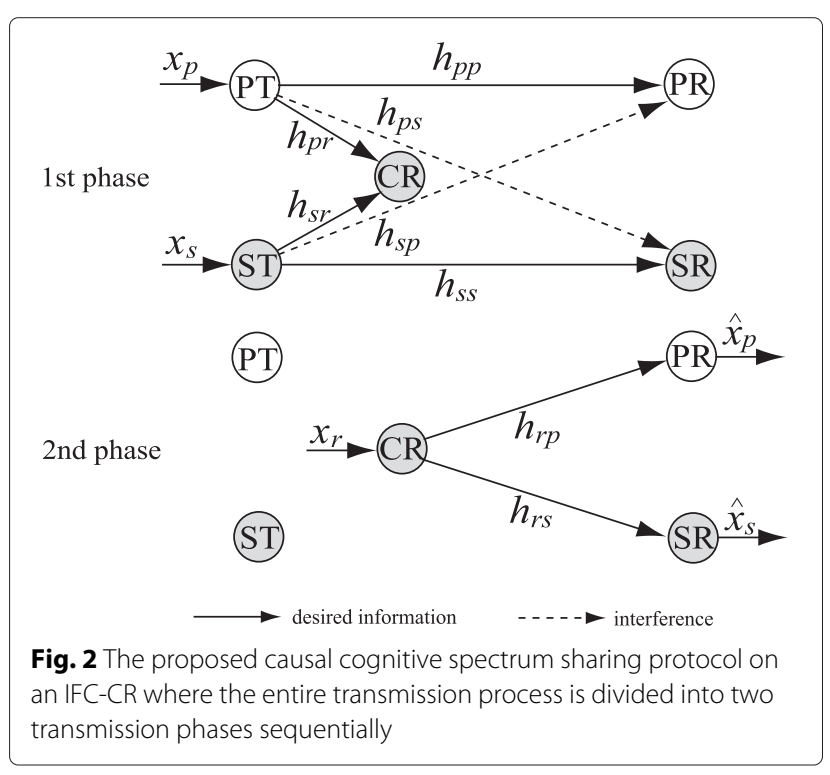


primary system, we assume that PR is only aware of a relay terminal but oblivious of the secondary system [20-23], thus the component of the secondary signal is simply treated as noise at PR [24-26]. This provides a performance lower bound for the primary system compared to the cases with sophisticated decoding strategies. On the other hand, at the secondary receiver (SR), by exploiting the received signals in two phases, SIC is employed to decode the desired message. The contributions of this paper are summarized as follows.

- In decoding the mixed signals using SIC, we define an event to describe whether a specific message can be successfully recovered. In order to illustrate the correlation between the successive events in decoding mixed signals, we introduce a graphical representation by which each event can be represented by the corresponding region in a 2dimensional (or 3-dimensional) graph. On this basis, by integrating over the respective regions of events, accurate closed-form expressions of the end-to-end outage probability can be derived for both primary and secondary users under the proposed protocol.

- Without requiring non-causal knowledge, CR attempts to decode both primary and secondary messages after a first transmission phase in the proposed protocol. For the case where both messages are successfully recovered at CR after the first transmission phase, in order to further mitigate the mutual interference, we propose using DPC at CR to pre-cancel the interference seen at PR or SR in the subsequent relaying phase. Numerical results demonstrate a performance upper bound for the primary (or secondary) user, without affecting the performance of the other user.

- To guarantee that no harm is caused to the primary system, besides the power allocation performed at CR to forward the primary and secondary messages respectively, we find that a power control at ST is also needed to facilitate the SIC decoding at CR as well as to limit the interference caused to PR. Numerical results demonstrate that with a proper design of the power allocation at CR and the transmit power at ST, the secondary user is allowed to access the licensed spectrum and at the same time performance gains can be achieved for both primary and secondary systems.

The rest of this paper is organized as follows. Section 2 describes the system model, where the two successive phases are discussed and the end-to-end outage probability of IFC-CR is defined. In Section 3, based on the possible decoding results at $C R$ in the first transmission phase, the corresponding performance at $P R$ and $\mathrm{SR}$ in the second phase is analyzed. By exploiting the decoded messages, in Section 4, we propose using DPC at CR, which provides a performance upper bound. Simulation results are presented in Section 5 where the effects of different parameters are evaluated. Finally, Section 6 concludes the paper.

\section{System model and protocol description}

As shown in Fig. 2, we consider an IFC-CR where a ST/SR pair co-exists with a PT/PR pair in the same frequency band with the assistance of a CR. It is assumed that all nodes operate in half-duplex mode and the channels experience independent block Rayleigh fading. For notational simplicity, we let $h_{p p}$ and $h_{s s}$ denote the coefficients of the direct channels from $\mathrm{PT} \rightarrow \mathrm{PR}$ and $\mathrm{ST} \rightarrow \mathrm{SR}$, let $h_{p s}$ and $h_{s p}$ denote the coefficients of the cross interfering channels from $\mathrm{PT} \rightarrow \mathrm{SR}$ and $\mathrm{ST} \rightarrow \mathrm{PR}$, and let $h_{p r}, h_{s r}, h_{r p}$, and $h_{r s}$ denote the coefficients of the relay channels from $\mathrm{PT} \rightarrow \mathrm{CR}, \mathrm{ST} \rightarrow \mathrm{CR}, \mathrm{CR} \rightarrow \mathrm{PR}$, and $\mathrm{CR} \rightarrow \mathrm{SR}$, respectively. Then, we have the channel coefficient $h_{i j} \sim \mathcal{C N}\left(0, \delta_{i j}^{-1}\right)$, where $i j \in\{p p, s s, p s, s p, p r, s r, r p, r s\}$ and $\delta_{i j}^{-1}$ denotes the corresponding average channel power gain. By letting $\gamma_{i j}=\left|h_{i j}\right|^{2}, \gamma_{i j}$ follows an exponential distribution that $\gamma_{i j} \sim \exp \left(\delta_{i j}\right)$ [34]. For ease of exposition, we assume that the additive white Gaussian noise (AWGN) $n_{0}$ is of zero mean and with unitary variance at each receiver. Any channel can be reduced to this normalized form. The transmit powers at PT, ST, and CR are denoted as $P_{P}$, $P_{S}$, and $P_{R}$, respectively. $x_{p}$ and $x_{s}$ denote the messages originated at PT and $\mathrm{ST}$, with target rates $R_{p t}$ and $R_{s t}$, respectively. For easy reference, we summarize the abbreviations, notations, and symbols that appear in this paper in Table 1.

As shown in Fig. 2, in order to maintain the causality of the system, we divide the entire transmission process into two phases as discussed in the following.

\subsection{First transmission phase}

In the first phase, both PT and ST transmit their respective messages simultaneously. Then, the corresponding received signal at $\mathrm{PR}, \mathrm{SR}$, and $\mathrm{CR}$ is given as

$$
y_{j}(1)=h_{p j} \sqrt{P_{P}} x_{p}+h_{s j} \sqrt{P_{S}} x_{s}+n_{0}, \quad j \in\{p, s, r\} .
$$

In order to recover $x_{p}$ and $x_{s}$ that are mixed together, SIC is performed at CR. Then, for the decoding results of $x_{p}$ and $x_{s}$, we define the following possible events that are mutually exclusive:

1. $\mathcal{E}^{(1)}=\left\{\right.$ Both $x_{p}$ and $x_{s}$ are successfully decoded at $\mathrm{CR}\}$;

2. $\mathcal{E}^{(2)}=\left\{\right.$ Only $x_{p}$ is successfully decoded at CR $\}$;

3. $\mathcal{E}^{(3)}=\left\{\right.$ Only $x_{s}$ is successfully decoded at $\left.\mathrm{CR}\right\}$;

4. $\mathcal{E}^{(4)}=\left\{\right.$ Neither of $x_{p}$ and $x_{s}$ is successfully decoded at $\mathrm{CR}\}$. 
Table 1 A summary of abbreviations, notations, and symbols

\begin{tabular}{|c|c|}
\hline $\mathrm{SIC}$ & Successive interference cancellation \\
\hline IFC-CR & Interference channel with a cognitive relay \\
\hline DPC & Dirty paper coding \\
\hline AF/DF & Amplify-and-forward/decode-and-forward \\
\hline PT/PR & Primary transmitter/primary receiver \\
\hline ST/SR & Secondary transmitter/secondary receiver \\
\hline MRC & Maximal-ratio combining \\
\hline AWGN & Additive white Gaussian noise \\
\hline$x_{p}$ & Signal transmitted from PT \\
\hline$x_{s}$ & Signal transmitted from ST \\
\hline$x_{r}$ & Signal transmitted from $C R$ \\
\hline$y_{p}$ & Signal received at PR \\
\hline$y_{s}$ & Signal received at SR \\
\hline$y_{r}$ & Signal received at $C R$ \\
\hline$n_{0}$ & AWGN that is with unitary variance \\
\hline$h_{i j}$ & Channel coefficient of link $i \rightarrow j$ \\
\hline$\gamma_{i j}=\left|h_{i j}\right|^{2}$ & Channel power gain of link $i \rightarrow j$ \\
\hline $\exp (\delta)$ & An exponential distribution with mean $\delta^{-1}$ \\
\hline$P_{p}$ & Transmit power at PT \\
\hline$P_{S}$ & Transmit power at ST \\
\hline$P_{R}$ & Transmit power at $C R$ \\
\hline$R_{p t}$ & Target rate at PT \\
\hline$R_{s t}$ & Target rate at ST \\
\hline $\mathcal{E}, \overline{\mathcal{E}}$ & An event and its complementary event \\
\hline $\operatorname{Pr}\{\mathcal{E}\}$ & Probability of event $\mathcal{E}$ \\
\hline$O_{P}$ & End-to-end outage probability of the primary system \\
\hline Os & End-to-end outage probability of the secondary system \\
\hline$\alpha$ & Power allocation factor at CR \\
\hline$\theta$ & Ratio between $P_{S}$ and $P_{P}$ \\
\hline$\tau$ & Ratio between $\delta_{s r}^{-1}$ and $\delta_{p r}^{-1}$ \\
\hline$\varphi$ & Ratio between $\delta_{s p}^{-1}$ and $\delta_{p p}^{-1}$ \\
\hline
\end{tabular}

The corresponding probabilities are defined as $\operatorname{Pr}\left\{\mathcal{E}^{(1)}\right\}$, $\operatorname{Pr}\left\{\mathcal{E}^{(2)}\right\}, \operatorname{Pr}\left\{\mathcal{E}^{(3)}\right\}$, and $\operatorname{Pr}\left\{\mathcal{E}^{(4)}\right\}$ that will be derived in Section 3.1.

\subsection{Second transmission phase}

When at least one of $x_{p}$ and $x_{s}$ is successfully decoded by $\mathrm{CR}$, a power allocation is performed to forward a linear weighted combination of $x_{p}$ and $x_{s}$ in the second phase. Otherwise, CR simply stays silent and both PT and ST perform retransmissions simultaneously.

\subsubsection{Conditioned on event $\mathcal{E}^{(1)}$}

In the second phase, $\mathrm{CR}$ broadcasts a composite message

$$
x_{r}=\sqrt{\alpha P_{R}} x_{p}+\sqrt{(1-\alpha) P_{R}} x_{s},
$$

where $\alpha \in(0,1)$ is the power allocation factor for relaying the primary message $x_{p}$. Then, the corresponding received signal at PR and SR is given as

$$
y_{j}(2)=h_{r j} \sqrt{\alpha P_{R}} x_{p}+h_{r j} \sqrt{(1-\alpha) P_{R}} x_{s}+n_{0}, \quad j \in\{p, s\} .
$$

\subsubsection{Conditioned on event $\mathcal{E}^{(2)}$}

In the second phase, $\mathrm{CR}$ broadcasts a composite message

$$
\begin{aligned}
x_{r} & =\sqrt{\alpha P_{R}} x_{p}+\beta\left(h_{s r} \sqrt{P_{S}} x_{s}+n_{0}\right), \text { where } \\
\beta & =\sqrt{\frac{(1-\alpha) P_{R}}{\gamma_{s r} P_{S}+1}} .
\end{aligned}
$$

Then, the corresponding received signal at $\mathrm{PR}$ and $\mathrm{SR}$ is given as

$$
\begin{aligned}
y_{j}(2)= & h_{r j} \sqrt{\alpha P_{R}} x_{p}+h_{r j} \beta\left(h_{s r} \sqrt{P_{S}} x_{s}+n_{0}\right) \\
& +n_{0}, \quad j \in\{p, s\} .
\end{aligned}
$$

\subsubsection{Conditioned on event $\mathcal{E}^{(3)}$}

In the second phase, $\mathrm{CR}$ broadcasts a composite message

$$
\begin{aligned}
x_{r} & =\beta\left(h_{p r} \sqrt{P_{P}} x_{p}+n_{0}\right)+\sqrt{(1-\alpha) P_{R}} x_{s}, \quad \text { where } \\
\beta & =\sqrt{\frac{\alpha P_{R}}{\gamma_{p r} P_{P}+1}} .
\end{aligned}
$$

Then, the corresponding received signal at $\mathrm{PR}$ and $\mathrm{SR}$ is given as

$$
\begin{aligned}
y_{j}(2)= & h_{r j} \beta\left(h_{p r} \sqrt{P_{P}} x_{p}+n_{0}\right) \\
& +h_{r j} \sqrt{(1-\alpha) P_{R}} x_{s}+n_{0}, \quad j \in\{p, s\} .
\end{aligned}
$$

\subsubsection{Conditioned on event $\mathcal{E}^{(4)}$}

CR simply stays silent and PT and ST retransmit $x_{p}$ and $x_{s}$ respectively in the second phase. Then, the corresponding received signal at $\mathrm{PR}$ and $\mathrm{SR}$ is given as

$$
y_{j}(2)=h_{p j} \sqrt{P_{P}} x_{p}+h_{s j} \sqrt{P_{S}} x_{s}+n_{0}, \quad j \in\{p, s\} .
$$

\subsection{End-to-end performance}

For the decoding at PR at the end of the second phase, MRC is performed to decode $x_{p}$ by utilizing the received signals in two successive phases, i.e., $y_{p}(1)$ and $y_{p}(2)$, while treating the secondary component of $x_{s}$ simply as noise. Then, depending on the decoding results $\mathcal{E}^{(1)}, \mathcal{E}^{(2)}, \mathcal{E}^{(3)}$, and $\mathcal{E}^{(4)}$, at $\mathrm{CR}$ at the end of the first phase, we define $O_{P}^{(1)}$, $O_{P}^{(2)}, O_{P}^{(3)}$, and $O_{P}^{(4)}$ as the corresponding outage probabilities at PR at the end of the second phase. On the other hand, SIC is performed at SR to decode the desired message $x_{s}$ by utilizing both received signals $y_{s}(1)$ and $y_{s}(2)$. 
Similarly, we define $O_{S}^{(1)}, O_{S}^{(2)}, O_{S}^{(3)}$, and $O_{S}^{(4)}$ as the corresponding outage probabilities at $\mathrm{SR}$ at the end of the second phase.

Theorem 1. In the proposed spectrum sharing protocol on IFC-CR, taking into account all possible decoding results at $C R$ at the end of the first phase, the overall endto-end outage probabilities of the primary and secondary systems can be respectively derived as

$$
\begin{aligned}
O_{P}= & \operatorname{Pr}\left\{\mathcal{E}^{(1)}\right\} O_{P}^{(1)}+\operatorname{Pr}\left\{\mathcal{E}^{(2)}\right\} O_{P}^{(2)}+\operatorname{Pr}\left\{\mathcal{E}^{(3)}\right\} O_{P}^{(3)} \\
& +\operatorname{Pr}\left\{\mathcal{E}^{(4)}\right\} O_{P}^{(4)} \\
O_{S}= & \operatorname{Pr}\left\{\mathcal{E}^{(1)}\right\} O_{S}^{(1)}+\operatorname{Pr}\left\{\mathcal{E}^{(2)}\right\} O_{S}^{(2)}+\operatorname{Pr}\left\{\mathcal{E}^{(3)}\right\} O_{S}^{(3)} \\
& +\operatorname{Pr}\left\{\mathcal{E}^{(4)}\right\} O_{S}^{(4)} .
\end{aligned}
$$

Next, we proceed to analyze the decoding performance at $\mathrm{CR}$ as well as $\mathrm{PR}$ and $\mathrm{SR}$ in two successive phases, respectively.

\section{Numerical analysis}

\subsection{Decoding performance at CR in the first phase}

At the end of the first phase, CR attempts to decode both $x_{p}$ and $x_{s}$ from $y_{r}(1)$ using SIC. From (1), if the power level of $x_{p}$ is higher than that of $x_{s}$, then CR attempts to decode $x_{p}$ first by considering the component of $x_{s}$ as noise. Defining $C(\Lambda)=\frac{1}{2} \log _{2}(1+\Lambda)$, the achievable rate of $x_{p}$ is given as

$$
R_{1, p}=C\left(\frac{P_{P} \gamma_{p r}}{P_{S} \gamma_{s r}+1}\right)
$$

Thus, $x_{p}$ can be first decoded if event

$$
\mathcal{E}_{1, p}=\left\{R_{1, p} \geq R_{p t}\right\}=\left\{\gamma_{p r} \geq \frac{R_{p t}^{\prime} P_{S}}{P_{P}} \gamma_{s r}+\frac{R_{p t}^{\prime}}{P_{P}}\right\}
$$

occurs, where $R_{p t}^{\prime}=2^{2 R_{p t}}-1$. By reconstructing and removing the component of $x_{p}$ from $y_{r}(1)$, the achievable rate of the remaining $x_{s}$ is given as

$$
R_{2, s}=C\left(P_{S} \gamma_{s r}\right)
$$

which can be decoded successively if event

$$
\mathcal{E}_{2, s}=\left\{R_{2, s} \geq R_{s t}\right\}=\left\{\gamma_{s r} \geq \frac{R_{s t}^{\prime}}{P_{S}}\right\}
$$

occurs, where $R_{s t}^{\prime}=2^{2 R_{s t}}-1$.

Conversely, if the power level of $x_{s}$ is higher than that of $x_{p}$, then CR attempts to decode $x_{s}$ first by considering the component of $x_{p}$ as noise. Then, the achievable rate of $x_{s}$ that is subject to the interference from $x_{p}$ is given as

$$
R_{1, s}=C\left(\frac{P_{S} \gamma_{s r}}{P_{P} \gamma_{p r}+1}\right)
$$

which can be first decoded if event

$$
\mathcal{E}_{1, s}=\left\{R_{1, s} \geq R_{s t}\right\}=\left\{\gamma_{p r} \leq \frac{P_{S}}{R_{s t}^{\prime} P_{P}} \gamma_{s r}-\frac{1}{P_{P}}\right\}
$$

occurs. Similarly, by reconstructing and removing the component of $x_{s}$ from $y_{r}(1)$, the achievable rate of the remaining $x_{p}$ is given as

$$
R_{2, p}=C\left(P_{P} \gamma_{p r}\right)
$$

which can be decoded successively if event

$$
\mathcal{E}_{2, p}=\left\{R_{2, p} \geq R_{p t}\right\}=\left\{\gamma_{p r} \geq \frac{R_{p t}^{\prime}}{P_{P}}\right\}
$$

occurs.

Thus, from (12), (14), (16), and (18), all possible decoding results at $\mathrm{CR}$ using $\mathrm{SIC}$ can be expressed as

$$
\begin{aligned}
& \mathcal{E}^{(1)}=\left\{\left(\mathcal{E}_{1, p} \cap \mathcal{E}_{2, s}\right) \cup\left(\mathcal{E}_{1, s} \cap \mathcal{E}_{2, p}\right)\right\}, \\
& \mathcal{E}^{(2)}=\left\{\mathcal{E}_{1, p} \cap \overline{\mathcal{E}}_{2, s}\right\}, \\
& \mathcal{E}^{(3)}=\left\{\mathcal{E}_{1, s} \cap \overline{\mathcal{E}}_{2, p}\right\}, \\
& \mathcal{E}^{(4)}=\left\{\overline{\mathcal{E}}_{1, p} \cap \overline{\mathcal{E}}_{1, s}\right\} .
\end{aligned}
$$

Take a close look at (12), (14), (16), and (18); since the multiple-access channels $h_{s r}$ and $h_{p r}$ are independent with each other, we can draw a 2-dimensional graph of $\gamma_{s r}$ and $\gamma_{p r}$ where the events defined in (19a)-(19d) are represented by their respective regions, as shown in Fig. 3. Here, we assume that $R_{p t}, R_{s t} \geq 1$ such that there is no intersection between the regions of events $\mathcal{E}_{1, p}$ and $\mathcal{E}_{1, s}$. With the respective regions defined by $\left\{\gamma_{s r}, \gamma_{p r}\right\}$, the corresponding probability of each event can thus be obtained by

$$
\begin{aligned}
& \iint_{\left\{\gamma_{s r}, \gamma_{p r}\right\}} f\left(\gamma_{s r}, \gamma_{p r}\right) d \gamma_{p r} d \gamma_{s r} \\
& =\int_{\left\{\gamma_{s r}\right\}} f\left(\gamma_{s r}\right)\left[\int_{\left\{\gamma_{p r}\right\}} f\left(\gamma_{p r}\right) d \gamma_{p r}\right] d \gamma_{s r},
\end{aligned}
$$

where $f\left(\gamma_{s r}\right)=\delta_{s r} e^{-\delta_{s r} \gamma_{s r}}$ and $f\left(\gamma_{p r}\right)=\delta_{p r} e^{-\delta_{p r} \gamma_{p r}}$ denote the respective probability density functions (PDF) of $\gamma_{s r}$ and $\gamma_{p r}$, and $f\left(\gamma_{s r}, \gamma_{p r}\right)=f\left(\gamma_{s r}\right) f\left(\gamma_{p r}\right)$ denotes the joint PDF [34]. 


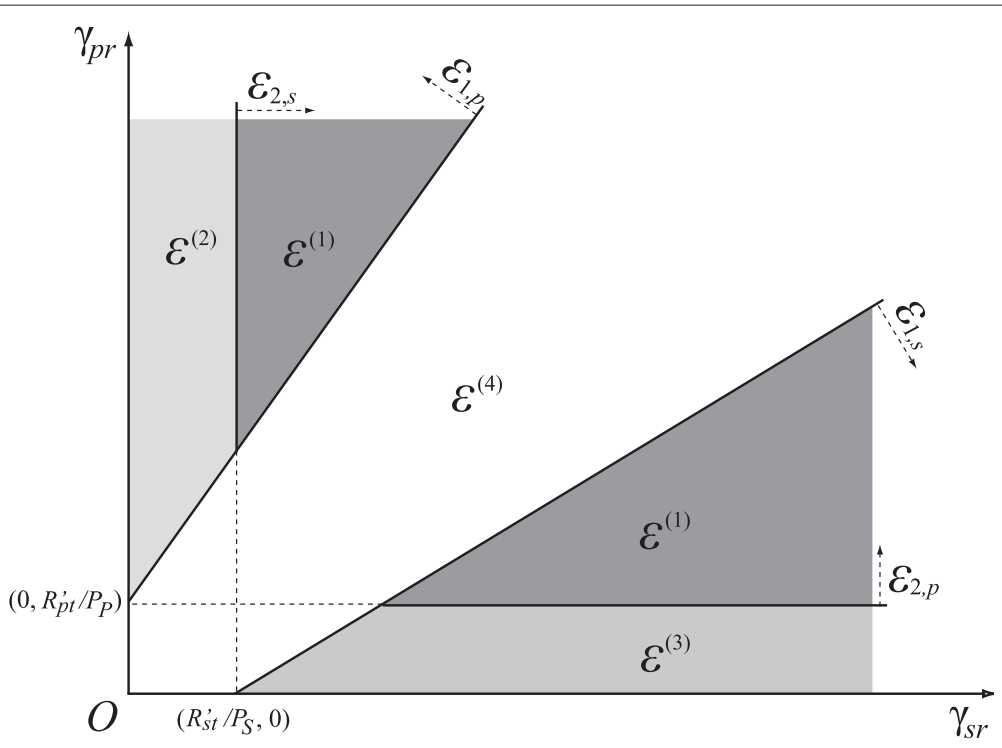

Fig. 3 Graphical representations of events $\mathcal{E}^{(1)}, \mathcal{E}^{(2)}, \mathcal{E}^{(3)}$, and $\boldsymbol{E}^{(4)}$ at $C R$ at the end of the first phase

Lemma 1. By employing SIC at CR to decode both $x_{p}$ and $x_{s}$, the respective probabilities of the events defined in (19a)-(19d) can be obtained as

$$
\begin{aligned}
\operatorname{Pr}\left\{\mathcal{E}^{(1)}\right\}= & \frac{\delta_{s r} e^{-\left(\frac{\delta_{s r} R_{s t}^{\prime}}{P_{S}}+\frac{\delta_{p r} R_{p t}^{\prime}\left(1+R_{s t}^{\prime}\right)}{P_{P}}\right)}}{\delta_{s r}+\frac{\delta_{p r} R_{p t}^{\prime} P_{S}}{P_{P}}} \\
+ & \frac{\delta_{p r} e^{-\left(\frac{\delta_{p r} R_{p t}^{\prime}}{P_{P}}+\frac{\delta_{s r} R_{s t}^{\prime}\left(1+R_{p t}^{\prime}\right)}{P_{S}}\right)}}{\delta_{p r}+\frac{\delta_{s r} R_{s t}^{\prime} P_{P}}{P_{S}}},
\end{aligned}
$$

$\operatorname{Pr}\left\{\mathcal{E}^{(2)}\right\}=\frac{\delta_{s r}\left[e^{-\frac{\delta_{p r} R_{p t}^{\prime}}{P_{P}}}-e^{-\left(\frac{\delta_{s r} R_{s t}^{\prime}}{P_{S}}+\frac{\delta_{p r} R_{p t}^{\prime}\left(1+R_{s t}^{\prime}\right)}{P_{P}}\right)}\right]}{\delta_{s r}+\frac{\delta_{p r} R_{p t}^{\prime} P_{S}}{P_{P}}}$

$\operatorname{Pr}\left\{\mathcal{E}^{(3)}\right\}=\frac{\delta_{p r}\left[e^{-\frac{\delta_{s r} R_{s t}^{\prime}}{P_{S}}}-e^{-\left(\frac{\delta_{p r} R_{p t}^{\prime}}{P_{P}}+\frac{\delta_{s r} R_{s t}^{\prime}\left(1+R_{p t}^{\prime}\right)}{P_{S}}\right)}\right]}{\delta_{p r}+\frac{\delta_{s r} R_{s t}^{\prime} P_{P}}{P_{S}}}$

$\operatorname{Pr}\left\{\mathcal{E}^{(4)}\right\}=1-\frac{\delta_{s r} e^{-\frac{\delta_{p r} R_{p t}^{\prime}}{P_{P}}}}{\delta_{s r}+\frac{\delta_{p r} R_{p t}^{\prime} P_{S}}{P_{P}}}-\frac{\delta_{p r} e^{-\frac{\delta_{s r} R_{s t}^{\prime}}{P_{S}}}}{\delta_{p r}+\frac{\delta_{s r} R_{s t}^{\prime} P_{P}}{P_{S}}}$.

Please find in Appendix A for the detailed derivations.

\subsection{Decoding performance at PR in the second phase}

\subsubsection{Conditioned on event $\mathcal{E}^{(1)}$}

Together with (1) and (3), MRC is performed at PR to decode the desired message $x_{p}$. Then, the corresponding achievable rate is given as

$$
\begin{aligned}
R_{p}^{(1)} & =C\left(\frac{P_{P} \gamma_{p p}}{P_{S} \gamma_{s p}+1}+\frac{\alpha}{1-\alpha+\frac{1}{P_{R} \gamma_{r p}}}\right) \\
& \approx C\left(\frac{P_{P} \gamma_{p p}}{P_{S} \gamma_{s p}+1}+\frac{\alpha}{1-\alpha}\right),
\end{aligned}
$$

where the approximation is obtained assuming $P_{R} \gg 1$ $[24,25]$.

Lemma 2. Conditioned on event $\mathcal{E}^{(1)}$ that both $x_{p}$ and $x_{s}$ are successfully decoded at $C R$ at the end of the first phase, the corresponding outage probability at PR at the end of the second phase can be derived as

$$
\begin{aligned}
& O_{P}^{(1)}=\operatorname{Pr}\left\{R_{p}^{(1)}<R_{p t}\right\} \\
& \approx\left\{\begin{array}{rl}
0, & \alpha \geq \frac{R_{p t}^{\prime}}{R_{p t}^{\prime}+1} \\
1-\frac{\delta_{s p} e^{-\frac{\delta_{p p}\left(R_{p t}^{\prime}-\frac{\alpha}{1-\alpha}\right)}{P_{P}}}}{\delta_{s p}+\frac{\left(R_{p t}^{\prime}-\frac{\alpha}{1-\alpha}\right) P_{S} \delta_{p p}}{P_{P}}}, & \alpha<\frac{R_{p t}^{\prime}}{R_{p t}^{\prime}+1}
\end{array} .\right.
\end{aligned}
$$

Please find in Appendix B for the detailed derivations. 


\subsubsection{Conditioned on event $\mathcal{E}^{(2)}$}

Together with (1) and (5), by employing MRC at PR, the achievable rate of $x_{p}$ is given as

$$
\begin{aligned}
R_{p}^{(2)} & =C\left(\frac{P_{P} \gamma_{p p}}{P_{S} \gamma_{s p}+1}+\frac{\alpha P_{R} \gamma_{r p}}{\gamma_{r p} \beta^{2}\left(P_{S} \gamma_{s r}+1\right)+1}\right) \\
& =C\left(\frac{P_{P} \gamma_{p p}}{P_{S} \gamma_{s p}+1}+\frac{\alpha}{(1-\alpha)+\frac{1}{P_{R} \gamma_{r p}}}\right),
\end{aligned}
$$

which is exactly the same (22). This is because in both cases, $x_{p}$ is successfully decoded and forwarded by CR with power $\alpha P_{R}$. Thus, the corresponding outage probability at PR at the end of the second phase is

$$
O_{P}^{(2)}=O_{P}^{(1)}
$$

\subsubsection{Conditioned on event $\mathcal{E}^{(3)}$}

Together with (1) and (7), by employing MRC at PR, the achievable rate of $x_{p}$ is given as

$$
\begin{aligned}
R_{p}^{(3)}= & C\left(\frac{P_{P} \gamma_{p p}}{P_{S} \gamma_{s p}+1}+\frac{\gamma_{r p} \beta^{2} P_{P} \gamma_{p r}}{(1-\alpha) P_{R} \gamma_{r p}+\gamma_{r p} \beta^{2}+1}\right) \\
= & C\left(\frac{P_{P} \gamma_{p p}}{P_{S} \gamma_{s p}+1}\right. \\
& \left.+\frac{\alpha}{(1-\alpha)+\frac{1}{P_{P} \gamma_{p r}}+\frac{1}{P_{R} \gamma_{r p}}+\frac{1}{P_{P} \gamma_{p r} P_{R} \gamma_{r p}}}\right) \\
\approx & C\left(\frac{P_{P} \gamma_{p p}}{P_{S} \gamma_{s p}+1}+\frac{\alpha}{1-\alpha}\right),
\end{aligned}
$$

where the approximation in (26) is obtained assuming $P_{P}, P_{R} \gg 1[24,25]$. Since (26) is of the same form as (22), the corresponding outage probability at $\mathrm{PR}$ at the end of the second phase is

$$
O_{P}^{(3)} \approx O_{P}^{(1)}
$$

\subsubsection{Conditioned on event $\mathcal{E}^{(4)}$}

Together with (1) and (8), by employing MRC at PR, the achievable rate of $x_{p}$ is given as

$$
R_{p}^{(4)}=C\left(\frac{2 P_{P} \gamma_{p p}}{P_{S} \gamma_{s p}+1}\right)
$$

Lemma 3. Conditioned on event $\mathcal{E}^{(4)}$ that neither of $x_{p}$ and $x_{s}$ is successfully decoded at CR at the end of the first phase, the corresponding outage probability at $P R$ at the end of the second phase can be derived as

$$
O_{P}^{(4)}=\operatorname{Pr}\left\{R_{p}^{(4)}<R_{p t}\right\}=1-\frac{\delta_{s p} e^{-\frac{\delta_{p p} R_{p t}^{\prime}}{2 P_{P}}}}{\delta_{s p}+\frac{\delta_{p p} R_{p t}^{\prime} P_{S}}{2 P_{P}}}
$$

Please find in Appendix $\mathrm{C}$ for the detailed derivations.

Substituting (23), (25), (27), and (29) into (9), we can thus obtain the end-to-end outage probability $O_{P}$ of the primary system.

Theorem 2. Take a close look at (23), (25), and (27), all $O_{P}^{(1)}, O_{P}^{(2)}$, and $O_{P}^{(3)}$ approach 0 when $\alpha \geq \frac{R_{p t}^{\prime}}{R_{p t}^{\prime}+1}$. Then, from (9), the term $\operatorname{Pr}\left\{\mathcal{E}^{(4)}\right\} O_{P}^{(4)}$ dominates the end-to-end outage probability $O_{P}$. In other words, the component of $\operatorname{Pr}\left\{\mathcal{E}^{(4)}\right\} O_{P}^{(4)}$ brings a lower bound to $O_{P}$. Defining $\alpha^{*}=$ $\frac{R_{p t}^{\prime}}{R_{p t}^{\prime}+1}$, the end-to-end outage performance of the primary system is thus optimized when $\alpha \geq \alpha^{*}$, i.e.,

$$
\begin{aligned}
& \arg \min _{\alpha}\left\{O_{P}\right\}=\left\{\alpha \mid \alpha \geq \alpha^{*}\right\}, \text { and } \\
& \min _{\alpha}\left\{O_{P}\right\} \geq \operatorname{Pr}\left\{\mathcal{E}^{(4)}\right\} O_{P}^{(4)} .
\end{aligned}
$$

Remark 1. From Theorem 2, CR may simply select a power allocation factor $\alpha=\alpha^{*}=\frac{R_{p t}^{\prime}}{R_{p t}^{\prime}+1}$ such that a reasonably good performance is achieved for the primary system, without requiring the CSI or other relevant information.

Remark 2. For comparison purposes, we consider a benchmark case for the primary system without spectrum sharing, where PT transmits to PR directly without a relay. Then, with target rate $R_{p t}$, the corresponding outage probability is

$$
O_{P}^{\prime}=1-e^{-\frac{\delta_{p p}\left(2^{R} p t-1\right)}{P_{P}}}
$$

The details are omitted here for the sake of brevity. Together with (9) and (31), in order to provide the primary system an incentive to participate in the spectrum sharing, the following condition has to be satisfied

$$
O_{P} \leq O_{P}^{\prime}
$$


Remark 3. From (30) and (31), if $\operatorname{Pr}\left\{\mathcal{E}^{(4)}\right\} O_{P}^{(4)} \leq O_{P}^{\prime}$, then it is possible to find a suitable power allocation factor $\alpha, e . g$., $\alpha \geq \alpha^{*}$, such that the condition in (32) is satisfied. If however, $\operatorname{Pr}\left\{\mathcal{E}^{(4)}\right\} O_{P}^{(4)}>O_{P}^{\prime}$, then the primary system experiences a performance loss compared to the benchmark case even when $\alpha \rightarrow 1$. Thus, in order to guarantee the performance of the legacy primary system, apart from selecting a proper power allocation factor $\alpha \geq \alpha^{*}$ at $C R$, the transmit power $P_{S}$ also needs to be properly designed.

For the power control of $P_{S}$, it is assumed that the statistical CSI of $h_{s p}$ and $h_{p p}$ is available at ST, which is a common assumption made in existing works [20-23]. Assuming that the channels are reciprocal, the CSI can be acquired at ST through a feedback channel from PR [35-37]. In addition, other relevant information is also required, i.e., $P_{P}$ and $R_{p t}$, which is usually inserted in the header of a packet that can be overheard by ST. With these information, both $\operatorname{Pr}\left\{\mathcal{E}^{(4)}\right\} O_{P}^{(4)} \leq O_{P}^{\prime}$ and $O_{P}^{\prime}$ can be estimated at ST. Furthermore, we assume that the probability $\operatorname{Pr}\left\{\mathcal{E}^{(4)}\right\}$ is available at ST through a feedback channel from CR. Thus, although it is intractable to analytically derive $P_{S}$ such that $O_{P} \leq O_{P}^{\prime}$, as long as a suitable $P_{S}$ is found to make sure that $\operatorname{Pr}\left\{\mathcal{E}^{(4)}\right\} O_{P}^{(4)} \leq$ $O_{P}^{\prime}$, it is possible to achieve cognitive spectrum sharing while proving a performance gain to the primary system.

\subsection{Decoding performance at SR in the second phase} 3.3.1 Conditioned on event $\mathcal{E}^{(1)}$

Together with (1) and (3), SR attempts to decode the desired message $x_{s}$ from the the mixed signals of $x_{s}$ and $x_{p}$ using SIC. Similar to (11), (13), and (15), we have the following achievable rates in decoding $x_{p}$ and $x_{s}$ successively

$$
\begin{aligned}
R_{1, p}^{(1)} & =C\left(\frac{P_{P} \gamma_{p s}}{P_{S} \gamma_{s s}+1}+\frac{\alpha}{1-\alpha+\frac{1}{P_{R} \gamma_{r s}}}\right) \\
& \approx C\left(\frac{P_{P} \gamma_{p s}}{P_{S} \gamma_{s s}+1}+\frac{\alpha}{1-\alpha}\right), \\
R_{2, s}^{(1)} & =C\left(P_{S} \gamma_{s s}+(1-\alpha) P_{R} \gamma_{r s}\right), \\
R_{1, s}^{(1)} & =C\left(\frac{P_{S} \gamma_{s s}}{P_{P} \gamma_{p s}+1}+\frac{1-\alpha}{\alpha+\frac{1}{P_{R} \gamma_{r s}}}\right) \\
& \approx C\left(\frac{P_{S} \gamma_{s s}}{P_{P} \gamma_{p s}+1}+\frac{1-\alpha}{\alpha}\right),
\end{aligned}
$$

where the approximations in (33a) and (33c) are obtained assuming $P_{R} \gg 1[24,25]$. Here, $R_{1, p}^{(1)}$ denotes the achievable rate of decoding $x_{p}$ directly by considering the component of $x_{s}$ simply as noise. Upon successfully decoding and removing $x_{p}, R_{2, s}^{(1)}$ denotes the achievable rate of decoding the remaining $x_{s}$ successively. Conversely, $R_{1, s}^{(1)}$ denotes the achievable rate of decoding $x_{s}$ directly that is subject to the interference from $x_{p}$. Then, similar to (12), (14), and (16), we have the corresponding events in decoding the mixed signals of $x_{p}$ and $x_{s}$ by using SIC

$$
\begin{aligned}
\mathcal{E}_{1, p}^{(1)} & =\left\{R_{1, p}^{(1)} \geq R_{p t}\right\} \\
& \approx\left\{\begin{array}{c}
\gamma_{p s} \geq \frac{\left(R_{p t}^{\prime}-\frac{\alpha}{1-\alpha}\right) P_{S}}{P_{P}} \gamma_{s s}+\frac{R_{p t}^{\prime}-\frac{\alpha}{1-\alpha}}{P_{P}}, \alpha<\frac{R_{p t}^{\prime}}{R_{p t}^{\prime}+1} \\
\text { certain event, } \quad \alpha \geq \frac{R_{p t}^{\prime}}{R_{p t}^{\prime}+1}
\end{array}\right. \\
\mathcal{E}_{2, s}^{(1)}=\left\{R_{2, s}^{(1)} \geq R_{s t}\right\} & \left\{\begin{array}{c}
\left.\gamma_{r s} \geq \frac{R_{s t}^{\prime}}{(1-\alpha) P_{R}}-\frac{P_{S}}{(1-\alpha) P_{R}} \gamma_{s s}\right\} \\
\mathcal{E}_{1, s}^{(1)}=\left\{R_{1, s}^{(1)} \geq R_{s t}\right\} \quad \alpha \leq \frac{1}{R_{s t}^{\prime}+1}
\end{array}\right. \\
& \approx\left\{\begin{array}{c}
\gamma_{p s} \leq \frac{P_{S}}{\left(R_{s t}^{\prime}-\frac{1-\alpha}{\alpha}\right) P_{P}} \gamma_{s s}-\frac{1}{P_{P}}, \alpha>\frac{1}{R_{s t}^{\prime}+1} \\
\text { certain event, } \quad
\end{array}\right.
\end{aligned}
$$

From (34), the event of successfully decoding $x_{s}$ by using SIC can thus be expressed as

$$
\mathcal{E}_{1, s}^{(1)} \cup\left(\overline{\mathcal{E}}_{1, s}^{(1)} \cap \mathcal{E}_{1, p}^{(1)} \cap \mathcal{E}_{2, s}^{(1)}\right)=\mathcal{E}_{1, s}^{(1)} \cup\left(\mathcal{E}_{1, p}^{(1)} \cap \mathcal{E}_{2, s}^{(1)}\right) .
$$

Lemma 4. Conditioned on event $\mathcal{E}^{(1)}$ that both $x_{p}$ and $x_{s}$ are successfully decoded at $C R$ at the end of the first phase, the corresponding outage probability at SR at the end of the second phase can be derived as

$$
\begin{aligned}
O_{S}^{(1)}= & -\operatorname{Pr}\left\{\mathcal{E}_{1, s}^{(1)}\right\}-\operatorname{Pr}\left\{\mathcal{E}_{1, p}^{(1)} \cap \mathcal{E}_{2, s}^{(1)}\right\} \\
& +\operatorname{Pr}\left\{\mathcal{E}_{1, p}^{(1)} \cap \mathcal{E}_{1, s}^{(1)}\right\},
\end{aligned}
$$




$$
\begin{aligned}
& \operatorname{Pr}\left\{\mathcal{E}_{1, s}^{(1)}\right\} \approx \begin{cases}\frac{\delta_{p s} e^{-\frac{\delta_{s s}\left(R_{s t}^{\prime}-\frac{1-\alpha}{\alpha}\right)}{P_{S}}}}{\delta_{p s}+\frac{\delta_{s s}\left(R_{s t}^{\prime}-\frac{1-\alpha}{\alpha}\right) P_{P}}{P_{S}}}, & \alpha>\frac{1}{R_{s t}^{\prime}+1}, \\
1, & \alpha \leq \frac{1}{R_{s t}^{\prime}+1}\end{cases}
\end{aligned}
$$

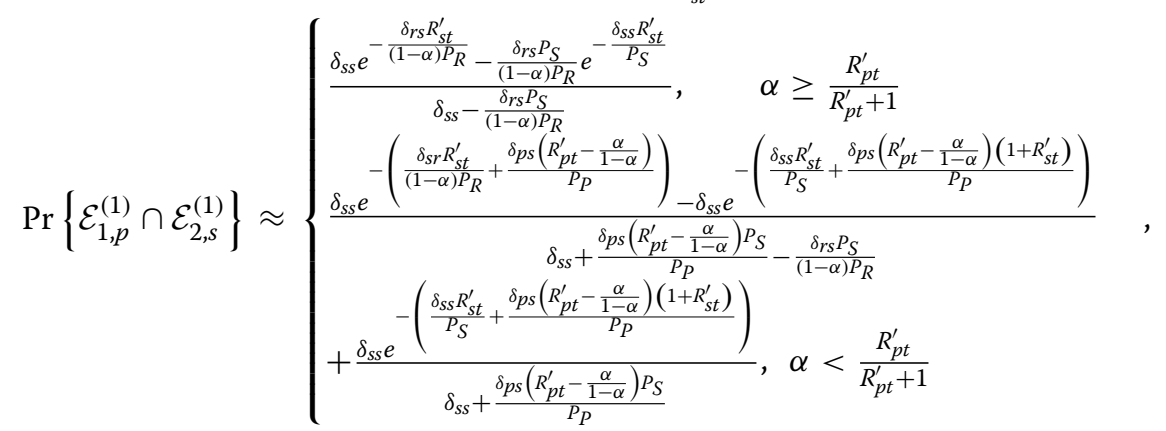

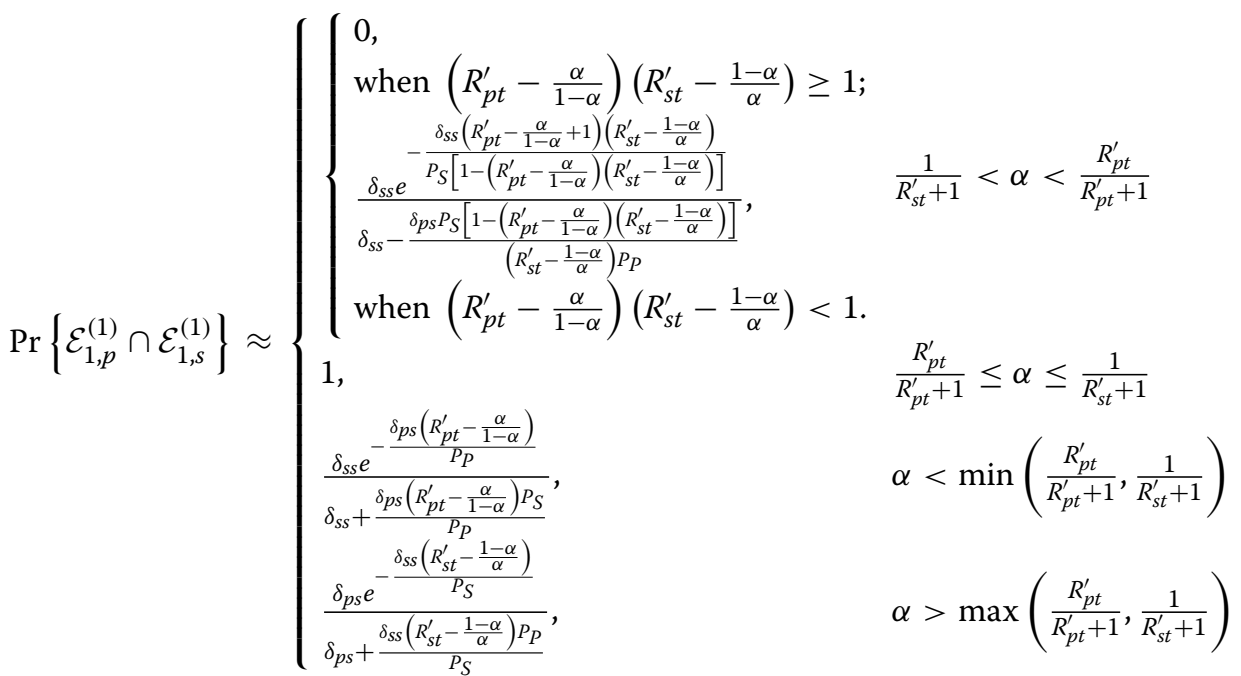

Please find in Appendix D for the detailed derivations. where

\subsubsection{Conditioned on event $\mathcal{E}^{(2)}$}

Together with (1) and (5), SR attempts to decode the desired message $x_{s}$ using SIC. Similarly, we have the following achievable rates in decoding $x_{p}$ and $x_{s}$ successively

$$
\begin{aligned}
R_{1, p}^{(2)} & =C\left(\frac{P_{P} \gamma_{p s}}{P_{S} \gamma_{s s}+1}+\frac{\alpha}{1-\alpha+\frac{1}{P_{R} \gamma_{r s}}}\right) \\
& \approx C\left(\frac{P_{P} \gamma_{p s}}{P_{S} \gamma_{s s}+1}+\frac{\alpha}{1-\alpha}\right) \\
R_{2, s}^{(2)} & =C\left(P_{S} \gamma_{s s}+\frac{(1-\alpha) P_{R} \gamma_{r s} P_{S} \gamma_{s r}}{(1-\alpha) P_{R} \gamma_{r s}+P_{S} \gamma_{s r}+1}\right) \\
& \approx C\left(P_{S} \gamma_{s s}+P_{S} \gamma_{s r}\right), \\
R_{1, s}^{(2)} & =C\left(\frac{P_{S} \gamma_{s s}}{P_{P} \gamma_{p s}+1}+\frac{1-\alpha}{\alpha+\frac{1}{P_{S} \gamma_{s r}}+\frac{1}{P_{R} \gamma_{r s}}+\frac{1}{P_{S} \gamma_{s r} P_{R} \gamma_{r s}}}\right) \\
& \approx C\left(\frac{P_{S} \gamma_{s s}}{P_{P} \gamma_{p s}+1}+\frac{1-\alpha}{\alpha}\right),
\end{aligned}
$$

where the approximation in (38a) is obtained assuming $P_{R} \gg 1$, the approximation in (38b) is obtained assuming $P_{R} \gamma_{r s} \gg P_{S} \gamma_{s r}$, and the approximation in (38c) is obtained assuming $P_{S}, P_{R} \gg 1[24,25]$, respectively. Then, we have the corresponding events in decoding $x_{p}$ and $x_{s}$ successively

$$
\begin{aligned}
& \mathcal{E}_{1, p}^{(2)}=\left\{R_{1, p}^{(2)} \geq R_{p t}\right\} \\
& \approx\left\{\begin{array}{cc}
\gamma_{p s} \geq \frac{\left(R_{p t}^{\prime}-\frac{\alpha}{1-\alpha}\right) P_{S}}{P_{P}} \gamma_{s s}+\frac{R_{p t}^{\prime}-\frac{\alpha}{1-\alpha}}{P_{P}}, \alpha<\frac{R_{p t}^{\prime}}{R_{p t}^{\prime}+1}, \\
\text { certainevent, }
\end{array}\right. \\
& \mathcal{E}_{2, s}^{(2)}=\left\{\begin{array}{c}
R_{p t}^{\prime} \\
R_{p t}^{\prime}+1
\end{array}\right.\left\{R_{2, s}^{(2)} \geq R_{s t}\right\}=\left\{\gamma_{s r} \geq \frac{R_{s t}^{\prime}}{P_{S}}-\gamma_{s s}\right\}, \\
& \mathcal{E}_{1, s}^{(2)}=\left\{R_{1, s}^{(2)} \geq R_{s t}\right\} \\
& \approx\left\{\begin{array}{c}
\gamma_{p s} \leq \frac{P_{S}}{\left(R_{s t}^{\prime}-\frac{1-\alpha}{\alpha}\right) P_{P}} \gamma_{s s}-\frac{1}{P_{P}}, \alpha>\frac{1}{R_{s t}^{\prime}+1} \\
\text { certainevent, } \quad \alpha \leq \frac{1}{R_{s t}^{\prime}+1}
\end{array}\right.
\end{aligned}
$$


Similarly, the outage probability at SR at the end of the second phase can be derived as

$$
\begin{aligned}
O_{S}^{(2)}= & 1-\operatorname{Pr}\left\{\mathcal{E}_{1, s}^{(2)}\right\}-\operatorname{Pr}\left\{\mathcal{E}_{1, p}^{(2)} \cap \mathcal{E}_{2, s}^{(2)}\right\} \\
& +\operatorname{Pr}\left\{\mathcal{E}_{1, p}^{(2)} \cap \mathcal{E}_{1, s}^{(2)}\right\} .
\end{aligned}
$$

From (34) and (39), since $\mathcal{E}_{1, p}^{(2)}$ and $\mathcal{E}_{1, s}^{(2)}$ are of the same form as $\mathcal{E}_{1, p}^{(1)}$ and $\mathcal{E}_{1, s}^{(1)}$ respectively, we have

$$
\begin{aligned}
\operatorname{Pr}\left\{\mathcal{E}_{1, s}^{(2)}\right\} & =\operatorname{Pr}\left\{\mathcal{E}_{1, s}^{(1)}\right\}, \\
\operatorname{Pr}\left\{\mathcal{E}_{1, p}^{(2)} \cap \mathcal{E}_{1, s}^{(2)}\right\} & =\operatorname{Pr}\left\{\mathcal{E}_{1, p}^{(1)} \cap \mathcal{E}_{1, s}^{(1)}\right\} .
\end{aligned}
$$

Then, by integrating over the corresponding region of event $\left\{\mathcal{E}_{1, p}^{(2)} \cap \mathcal{E}_{2, s}^{(2)}\right\}$, we can obtain

$$
\begin{aligned}
& \operatorname{Pr}\left\{\mathcal{E}_{1, p}^{(2)} \cap \mathcal{E}_{2, s}^{(2)}\right\} \\
& \approx\left\{\begin{array}{l}
\frac{\delta_{s s} e^{-\frac{\delta_{s r} R_{s t}^{\prime}}{P_{S}}}-\delta_{s r} e^{-\frac{\delta_{s s} R_{s t}^{\prime}}{P_{S}}},}{\delta_{s s}-\delta_{s r}}, \quad \alpha \geq \frac{R_{p t}^{\prime}}{R_{p t}^{\prime}+1} \\
\frac{\delta_{s s} e^{-\left(\frac{\delta_{s r} R_{s t}^{\prime}}{P_{S}}+\frac{\delta_{p s}\left(R_{p t}^{\prime}-\frac{\alpha}{1-\alpha}\right)}{P_{P}}\right)}{ }_{-\delta_{s s} e^{-}}-\left(\frac{\delta_{s s} R_{s t}^{\prime}}{P_{S}}+\frac{\delta_{p s}\left(R_{p t}^{\prime}-\frac{\alpha}{1-\alpha}\right)\left(1+R_{s t}^{\prime}\right)}{P_{P}}\right)}{\delta_{s s}+\frac{\delta_{p s}\left(R_{p t}^{\prime}-\frac{\alpha}{1-\alpha}\right) P_{S}}{P_{P}}-\delta_{s r}} \\
+\frac{\delta_{s s} e-\left(\frac{\delta_{s s} R_{s t}^{\prime}}{P_{S}}+\frac{\delta_{p s}\left(R_{p t}^{\prime}-\frac{\alpha}{1-\alpha}\right)\left(1+R_{s t}^{\prime}\right)}{P_{P}}\right)}{\delta_{s s}+\frac{\delta_{p s}\left(R_{p t}^{\prime}-\frac{\alpha}{1-\alpha}\right) P_{S}}{P_{P}}}, \quad \alpha<\frac{R_{p t}^{\prime}}{R_{p t}^{\prime}+1}
\end{array}\right.
\end{aligned}
$$

\subsubsection{Conditioned on event $\mathcal{E}^{(3)}$}

Together with (1) and (7), SR attempts to decode the desired message $x_{s}$ using SIC. Similarly, we have the following achievable rates in decoding $x_{p}$ and $x_{s}$ successively

$$
\begin{aligned}
R_{1, p}^{(3)}= & C\left(\frac{P_{P} \gamma_{p s}}{P_{S} \gamma_{s s}+1}\right. \\
& \left.+\frac{\alpha}{1-\alpha+\frac{1}{P_{P} \gamma_{p r}}+\frac{1}{P_{R} \gamma_{r s}}+\frac{1}{P_{P} \gamma_{p r} P_{R} \gamma_{r s}}}\right) \\
\approx & C\left(\frac{P_{P} \gamma_{p s}}{P_{S} \gamma_{s s}+1}+\frac{\alpha}{1-\alpha}\right) \\
R_{2, s}^{(3)}= & C\left(P_{S} \gamma_{s s}+(1-\alpha) P_{R} \gamma_{r s}\right) \\
R_{1, s}^{(3)}= & C\left(\frac{P_{S} \gamma_{s s}}{P_{P} \gamma_{p s}+1}+\frac{1-\alpha}{\alpha+\frac{1}{P_{R} \gamma_{r s}}}\right) \\
\approx & C\left(\frac{P_{S} \gamma_{s s}}{P_{P} \gamma_{p s}+1}+\frac{1-\alpha}{\alpha}\right)
\end{aligned}
$$

where the approximation in (44a) is obtained assuming $P_{P}, P_{R} \gg 1$ and the approximation in $(44 \mathrm{c})$ is obtained assuming $P_{R} \gg 1[24,25]$, respectively.
From (44) and (33), since in both cases $x_{s}$ is successfully decoded and forwarded by $\mathrm{CR}$ with power $(1-\alpha) P_{R}$, we have

$$
O_{S}^{(3)}=O_{S}^{(1)}
$$

\subsubsection{Conditioned on event $\mathcal{E}^{(4)}$}

Again, SIC is performed at SR to decode the desired message $x_{s}$ by exploiting the received signals in (1) and (8). Then, we have the following achievable rates in decoding $x_{p}$ and $x_{s}$ successively

$$
\begin{aligned}
& R_{1, p}^{(4)}=C\left(\frac{2 P_{P} \gamma_{p s}}{P_{S} \gamma_{s s}+1}\right), \\
& R_{2, s}^{(4)}=C\left(2 P_{S} \gamma_{s s}\right), \\
& R_{1, s}^{(4)}=C\left(\frac{2 P_{S} \gamma_{s s}}{P_{P} \gamma_{p s}+1}\right) .
\end{aligned}
$$

Correspondingly, we have the following events in decoding $x_{p}$ and $x_{s}$ successively

$$
\begin{aligned}
& \mathcal{E}_{1, p}^{(4)}=\left\{R_{1, p}^{(4)} \geq R_{p t}\right\}=\left\{\gamma_{p s} \geq \frac{R_{p t}^{\prime} P_{S}}{2 P_{P}} \gamma_{s s}+\frac{R_{p t}^{\prime}}{2 P_{P}}\right\}, \\
& \mathcal{E}_{2, s}^{(4)}=\left\{R_{2, s}^{(4)} \geq R_{s t}\right\}=\left\{\gamma_{s s} \geq \frac{R_{s t}^{\prime}}{2 P_{S}}\right\}, \\
& \mathcal{E}_{1, s}^{(4)}=\left\{R_{1, s}^{(4)} \geq R_{s t}\right\}=\left\{\gamma_{p s} \leq \frac{2 P_{S}}{R_{s t}^{\prime} P_{P}} \gamma_{s s}-\frac{1}{P_{P}}\right\} .
\end{aligned}
$$

For the considered scenario where $R_{p t}, R_{s t} \geq 1$, there is no intersection between events $\mathcal{E}_{1, p}^{(4)}$ and $\mathcal{E}_{1, s}^{(4)}$. Thus, from Lemma 4 , the corresponding outage probability at SR can be derived as

$$
\begin{aligned}
& O_{S}^{(4)}=1-\operatorname{Pr}\left\{\mathcal{E}_{1, s}^{(4)}\right\}-\operatorname{Pr}\left\{\mathcal{E}_{1, p}^{(4)} \cap \mathcal{E}_{2, s}^{(4)}\right\} \\
& =1-\int_{\frac{R_{s t}^{\prime}}{2 P_{S}}}^{\infty} \delta_{s s} e^{-\delta_{s s} \gamma_{s s}}\left[\int_{\frac{R_{p t}^{\prime} P_{S}}{2 P_{P}} \gamma_{s s}+\frac{R_{p t}^{\prime}}{2 P_{P}}}^{\infty} \delta_{p s} e^{-\delta_{p s} \gamma_{p s}} d \gamma_{p s}\right] d \gamma_{s s} \\
& -\int_{\frac{R_{s t}^{\prime}}{2 P_{S}}}^{\infty} \delta_{s s} e^{-\delta_{s s} \gamma_{s s}}\left[\int_{0}^{\frac{2 P_{S}}{R_{s t}^{\prime} P_{P}} \gamma_{s s}-\frac{1}{P_{P}}} \delta_{p s} e^{-\delta_{p s} \gamma_{p s}} d \gamma_{p s}\right] d \gamma_{s s} \\
& =1-\frac{\frac{2 \delta_{p s} P_{S}}{R_{s t}^{\prime} P_{P}} e^{-\frac{\delta_{s s} R_{s t}^{\prime}}{2 P_{S}}}}{\delta_{s s}+\frac{2 \delta_{p s} P_{S}}{R_{s t}^{\prime} P_{P}}}-\frac{\delta_{s s} e^{-\left(\frac{\delta_{p s} R_{p t}^{\prime}}{2 P_{P}}+\frac{\delta_{s s} R_{s t}^{\prime}}{2 P_{S}}+\frac{\delta_{p s} R_{p t}^{\prime} R_{s t}^{\prime}}{4 P_{P}}\right)}}{\delta_{s s}+\frac{\delta_{p s} R_{p t}^{\prime} P_{s}}{2 P_{P}}},
\end{aligned}
$$

where $\delta_{s s} e^{-\delta_{s s} \gamma_{s s}}$ and $\delta_{p s} e^{-\delta_{p s} \gamma_{p s}}$ denote the respective PDFs of $\gamma_{s s}$ and $\gamma_{p s}$.

Substituting $O_{S}^{(1)}, O_{S}^{(2)}, O_{S}^{(3)}$, and $O_{S}^{(4)}$ into (10), we can thus obtain the overall end-to-end outage probability of the secondary system. 


\section{A performance upper bound}

Conditioned on event $\mathcal{E}^{(1)}$ that both $x_{p}$ and $x_{s}$ are successfully decoded at CR at the end of the first phase, we propose using DPC at CR to pre-cancel the interference seen at $\mathrm{SR}$ (or PR) in the second phase.

\subsection{A performance upper bound for secondary user}

We propose using DPC [17] at CR that transmits a composite message

$$
x_{r}^{\prime}=\sqrt{\alpha P_{R}} x_{p}+x_{s}^{\prime}
$$

in the second phase. Employing a similar coding scheme as in [26, Eq. (15)], $x_{s}^{\prime}$ is encoded using DPC by treating the component of $\sqrt{\alpha P_{R}} x_{p}$ as the known interference that will corrupt the reception at SR in the second phase. Thus, after dirty paper decoding, the effectively received signal at $\mathrm{SR}$ in the second phase is given as

$$
y_{s}^{\prime}(2)=h_{r s} \sqrt{(1-\alpha) P_{R}} x_{s}+n_{0} .
$$

That is, SR sees no interference in the second phase. Then, together with (1) and (50), SR attempts to decode $x_{s}$ using SIC. Following the same steps as in Lemma 4, the corresponding outage probability at SR at the end of the second phase can be similarly derived. The details are omitted here for the sake of brevity.

On the other hand, with the same power $\alpha P_{R}$ allocated to forward $x_{p}$ at $\mathrm{CR}$, the outage performance of the primary user is the same as that in (23).

\subsection{A performance upper bound for primary user}

The interference seen at PR can be also pre-cancelled by performing DPC at SR, where a composite message

$$
x_{r}^{\prime}=x_{p}^{\prime}+\sqrt{(1-\alpha) P_{R}} x_{s}
$$

is transmitted in the second phase. Similarly, $x_{p}^{\prime}$ is encoded using DPC by treating the component of $\sqrt{(1-\alpha) P_{R}} x_{s}$ as the known interference that will corrupt the reception at PR. Thus, after dirty paper decoding, the effectively received signal at $\mathrm{PR}$ in the second phase is given as

$$
y_{p}^{\prime}(2)=h_{r p} \sqrt{\alpha P_{R}} x_{p}+n_{0} .
$$

That is, PR sees no interference in the second phase. Again, together with (1) and (52), MRC is employed at PR to decode $x_{p}$. The details are omitted here for the sake of brevity.

On the other hand, with the same power $(1-\alpha) P_{R}$ allocated to forward $x_{s}$ at $\mathrm{CR}$, the outage performance of the secondary user is the same as that in (36).

Remark 4. From the above analysis, with a DPC performed at CR to exploit the successfully decoded messages received in the first phase, the interference seen at $S R$ (or PR) in the second phase can be pre-cancelled, thus obtaining a performance upper bound for the secondary (or primary) user without affecting the performance of the other user.

\section{Simulation results}

In this section, we illustrate the outage performance of both primary and secondary users in the proposed spectrum sharing protocol. In order to limit the interference caused to the primary user, we consider a power control at ST where $P_{S}=\theta P_{P}$. To evaluate the SIC decoding at $\mathrm{CR}$ at the end of the first phase, we let $\delta_{s r}^{-1}=\tau \delta_{p r}^{-1}$ for the multiple-access channels $h_{s r}$ and $h_{p r}$ at CR. To evaluate the interference seen at PR due to cross talk, we let $\delta_{s p}^{-1}=\varphi \delta_{p p}^{-1}$ for the multiple-access channels $h_{s p}$ and $h_{p p}$ at PR. Unless otherwise specified, we let $P_{P}=30 \mathrm{~dB}$, $P_{S}=10 \mathrm{~dB}, P_{R}=40 \mathrm{~dB}$, and $R_{s t}=R_{p t}=1$. In order to reflect the geometric structure of the considered network shown in Fig. 2, we let $\delta_{p p}^{-1}=\delta_{s s}^{-1}=0 \mathrm{~dB}$ for the direct links, $\delta_{s p}^{-1}=\delta_{p s}^{-1}=-10 \mathrm{~dB}$ for the cross links, and $\delta_{p r}^{-1}=$ $\delta_{s r}^{-1}=\delta_{r p}^{-1}=\delta_{r s}^{-1}=10 \mathrm{~dB}$ for the relay links, respectively. Simulation results are presented in Figs. 4, 5, 6, 7, 8, 9, 10, and 11 where lines denote the analytical results obtained in this paper and markers denote the results of Monte Carlo simulations.

\subsection{SIC decoding at CR}

From Theorem 2, in order to fully exploit the relay transmissions, event $\mathcal{E}^{(4)}$ that neither of $x_{p}$ and $x_{s}$ is decoded at $\mathrm{CR}$ should be avoided as much as possible. Figure 4 displays the probability of $\operatorname{Pr}\left\{\mathcal{E}^{(4)}\right\}$ with respect to $\theta$ where $P_{S}=\theta P_{P}$. Various channel conditions are considered where $\delta_{p r}^{-1}=10 \mathrm{~dB}$ and $\delta_{s r}^{-1}=\tau \delta_{p r}^{-1}$. As can be seen from Fig. 4, with an increase in $\theta$, the probability of $\operatorname{Pr}\left\{\mathcal{E}^{(4)}\right\}$ first increases and then decreases. This is reasonable as when there is a significant difference between the power levels of $x_{p}$ and $x_{s}$ received at CR, e.g., $\tau \theta \ll 1$ or $\tau \theta \gg 1$,

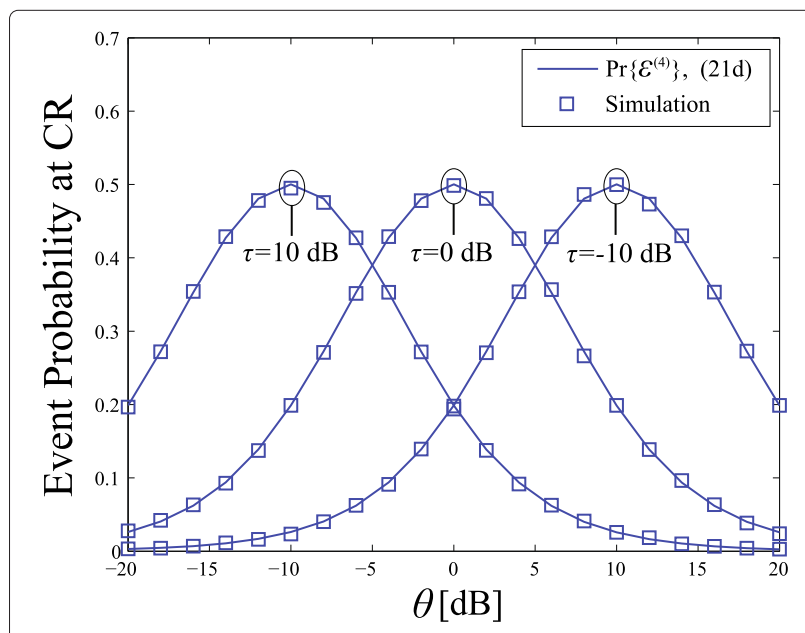

Fig. 4 The probability $\operatorname{Pr}\left\{\mathcal{E}^{(4)}\right\}$ with respect to $\theta$ where $P_{P}=30 \mathrm{~dB}$ and $P_{S}=\theta P_{p}$, when $\delta_{p r}^{-1}=10 \mathrm{~dB}$ and $\delta_{s r}^{-1}=\tau \delta_{p r}^{-1}$ 


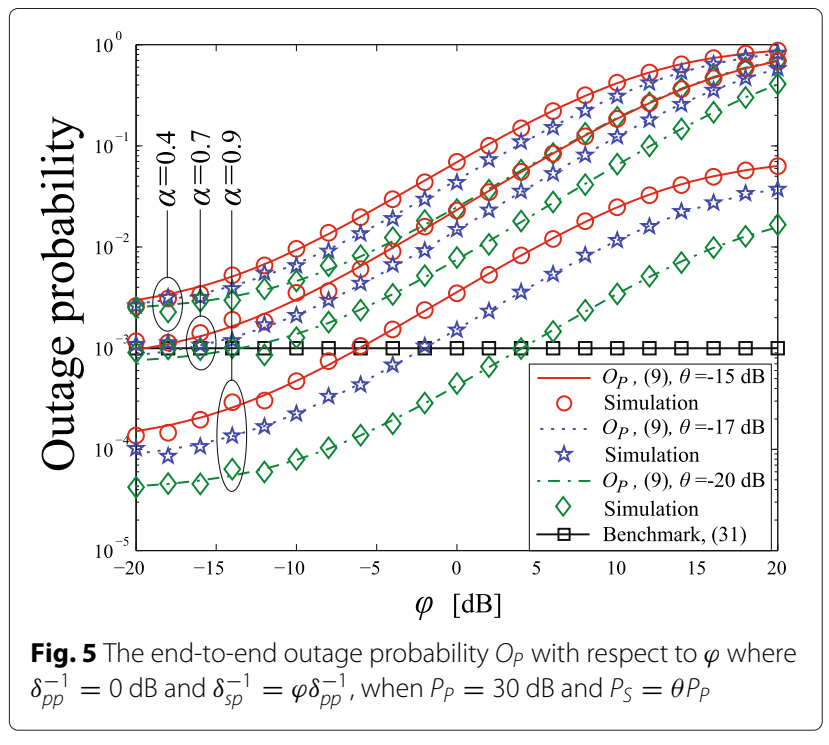

SIC is facilitated and it would be easy to decode $x_{p}$ and $x_{s}$ successively. In contrast, if the components of $x_{p}$ and $x_{s}$ are of comparable power levels, e.g., $\tau \theta \approx 1$, then the SIC decoding is limited by the mutual interference and it would be difficult to decode either of $x_{p}$ and $x_{s}$. This can be observed in Fig. 4 where $\operatorname{Pr}\left\{\mathcal{E}^{(4)}\right\}$ takes peak values at $\theta=-10,0,10 \mathrm{~dB}$ for $\tau=10,0,-10 \mathrm{~dB}$, respectively.

\subsection{End-to-end outage performance of the primary user}

Firstly, we evaluate how the interference due to cross talk affects the end-to-end performance of the primary system. Let $\delta_{s p}^{-1}=\varphi \delta_{p p}^{-1}, O_{P}$ is plotted with respect to $\varphi$ in Fig. 5 . The outage probability $O_{P}^{\prime}$ of the benchmark case considered in Remark 1 is also demonstrated. With an increase in $\varphi$, since the interference link ST $\rightarrow$ PR becomes stronger, the corresponding performance of the primary system is impaired. On the other hand, with an increase in $\theta$, ST transmits at a higher power that impedes the SIC decoding at CR as well as cause more interference to PR, thus similarly impairing the performance of the primary system. In addition, it is observed that a performance improvement is achieved for the primary system with a higher power allocation factor $\alpha$. When $\alpha=0.4$, even though ST transmits at a low power, e.g., $\theta=-20$ $\mathrm{dB}$, and the interference link $\mathrm{ST} \rightarrow \mathrm{PR}$ is very weak, e.g., $\varphi=-20 \mathrm{~dB}$, the primary user experiences a performance loss, i.e., $O_{P}>O_{P}^{\prime}$. Whereas when $\alpha=0.9$, with all other parameters being the same, a significant performance improvement is achieved for the primary system. This means that besides the power control at ST, the power allocation at $\mathrm{CR}$ also needs to be designed to compensate the interference caused to the primary system due to secondary transmissions.

To further illustrate the effects of $P_{S}$ and power allocation factor $\alpha, O_{P}$ is plotted with respect to $\theta$ and $\alpha$ in Fig. 6 . The outage probability $O_{P}^{\prime}$ of the benchmark case (31) is also illustrated. It is observed that when $\theta$ takes values smaller than 0.05 AND when $\alpha$ takes values greater than 0.75 , even subject to the interference from the secondary transmissions, the condition in (32) is always satisfied and a performance gain is achieved for the primary system. Otherwise, if $\theta>0.05$, then we have $O_{P}>O_{P}^{\prime}$ even when $\alpha \rightarrow 1$.

This can be better observed in Fig. 7 where a crosssectional view of Fig. 6 is demonstrated. With an increase in $\theta$, since the SIC at CR is impeded meanwhile more interference is caused to PR, the corresponding outage performance of the primary user is degraded. When $\theta=-20,-15 \mathrm{~dB}$, it is observed that we can always find

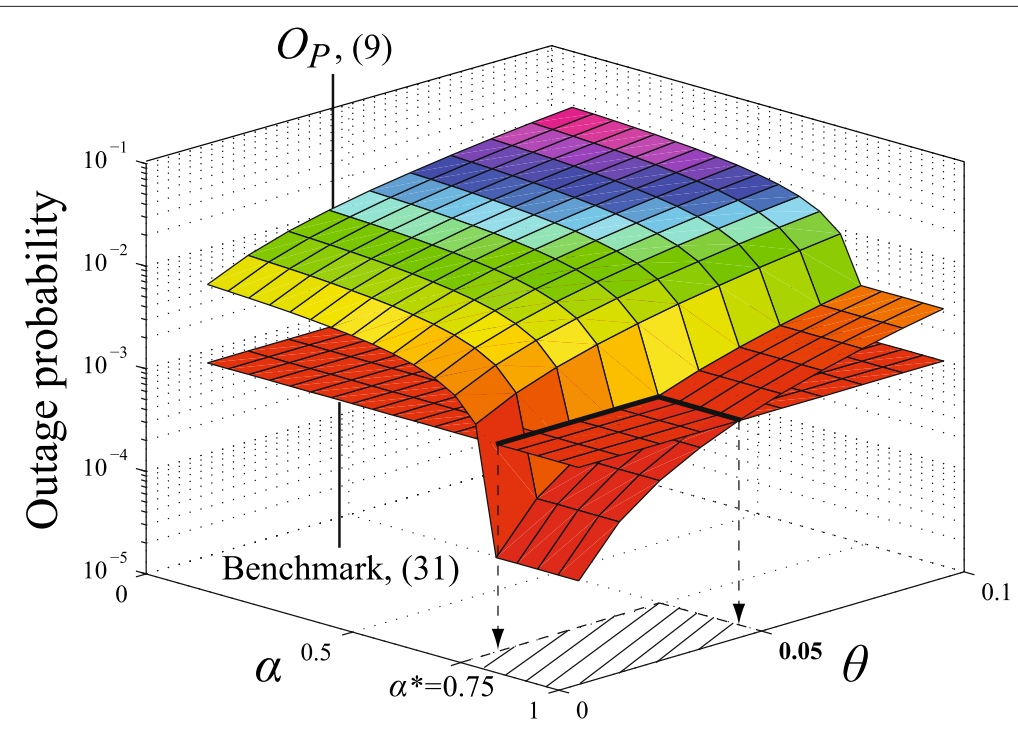

Fig. 6 The end-to-end outage probability $O_{P}$ with respect to $\alpha$ and $\theta$ where $P_{P}=30 \mathrm{~dB}$ and $P_{S}=\theta P_{P}$ 


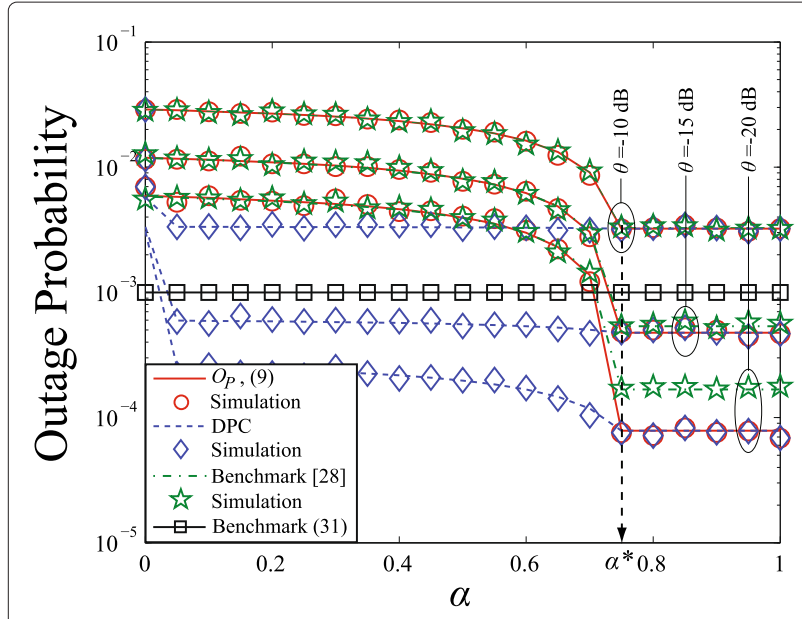

Fig. 7 A cross-sectional view of Fig. 6 when $\theta=-10,-15,-20$ $\mathrm{dB}$, respectively

a suitable power allocation factor $\alpha \geq \alpha^{*}=\frac{R_{p t}^{\prime}}{R_{p t}^{\prime}+1}=$ 0.75 such that a performance gain is achieved for the primary system. However, when $\theta$ is increased to $-10 \mathrm{~dB}$, it is observed that $O_{P}>O_{P}^{\prime}$ even when $\alpha \rightarrow 1$. Similar phenomena can be observed for a benchmark case considered in [28], where a linear weighted combination of primary and secondary messages is forwarded by CR only when both messages are successfully decoded. Otherwise, CR simply stays silent and both PT and ST perform a retransmission in the second phase. It is observed that with the same system parameters, a better performance is achieved by the proposed approach compared to that in [28]. This is reasonable as in the proposed approach, CR

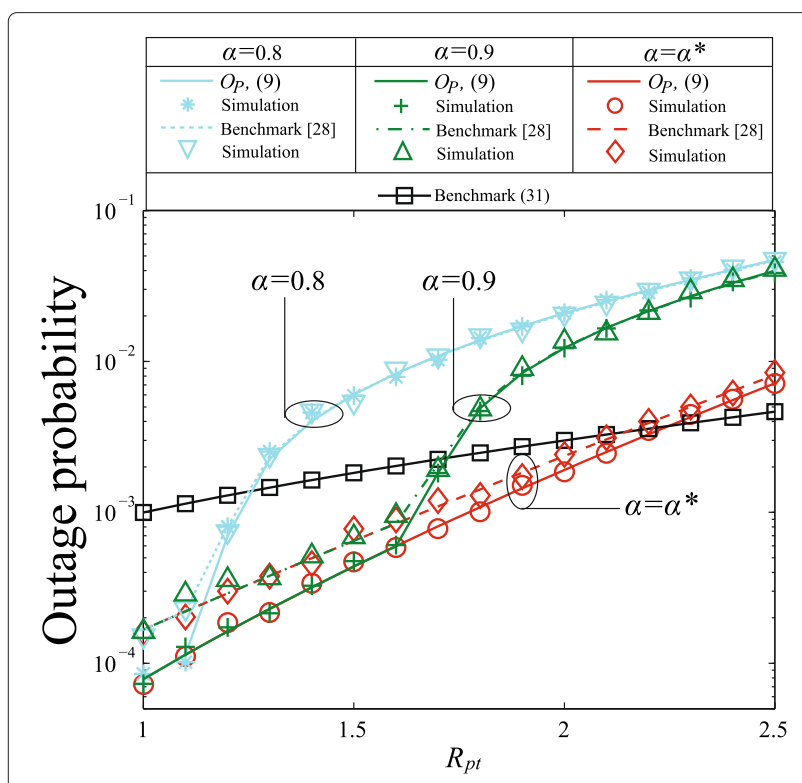

Fig. 8 The end-to-end outage probability $O_{p}$ with respect to $R_{p t}$ where $P_{S}=10 \mathrm{~dB}$ and $R_{S t}=1$

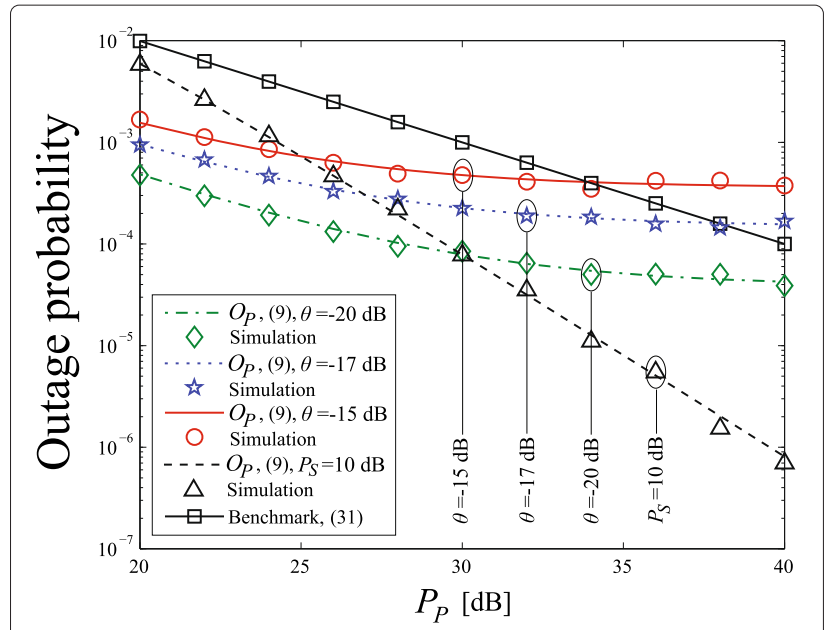

Fig. 9 The end-to-end outage probability $O_{P}$ with respect to $P_{P}$ where $\alpha=\alpha^{*}$

is able to help forward the received messages more frequently, which is more beneficial compared to a retransmission by PT and ST simultaneously that will cause severe interference to one another.

Furthermore, from Figs. 6 and 7, it is observed that when $\alpha$ takes values greater than $\alpha^{*}=\frac{R_{p t}^{\prime}}{R_{p t}^{\prime}+1}=0.75, O_{P}$ experiences a floor. This validates Theorem 2 that when $\alpha \geq \alpha^{*}$, $O_{P}^{(1)}, O_{P}^{(2)}$, and $O_{P}^{(3)}$ all approach 0 and thus $\operatorname{Pr}\left\{\mathcal{E}^{(4)}\right\} O_{P}^{(4)}$, which is irrelevant to $\alpha$, dominates the overall outage performance. For a better illustration, the outage performance using DPC at CR is also presented in Fig. 7. Conditioned on event $\mathcal{E}^{(1)}$ that both $x_{p}$ and $x_{s}$ are successfully decoded at CR, by using DPC to pre-cancel the interference seen at PR, it is observed that a performance upper bound is achieved for the primary system.

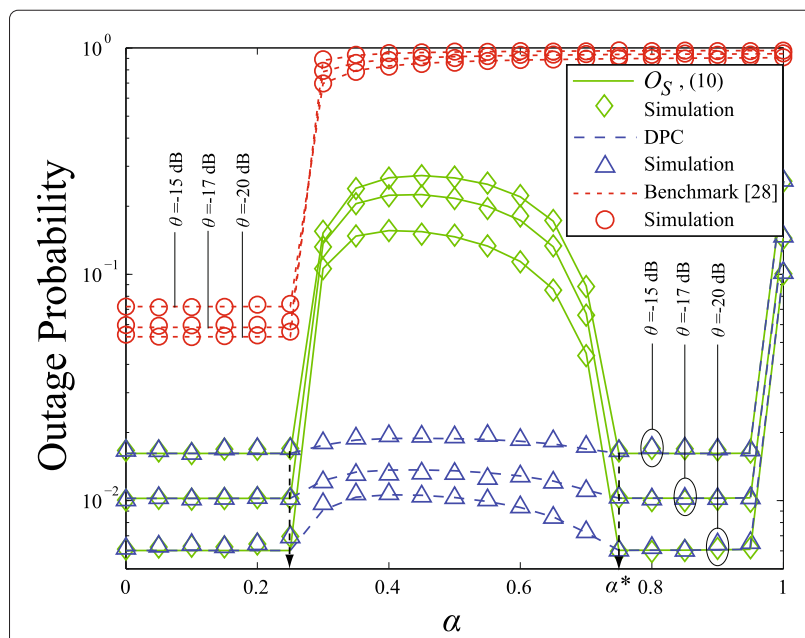

Fig. 10 The end-to-end outage probability $O_{S}$ with respect to $\alpha$ where $P_{p}=30 \mathrm{~dB}$ and $P_{S}=\theta P_{P}$ 


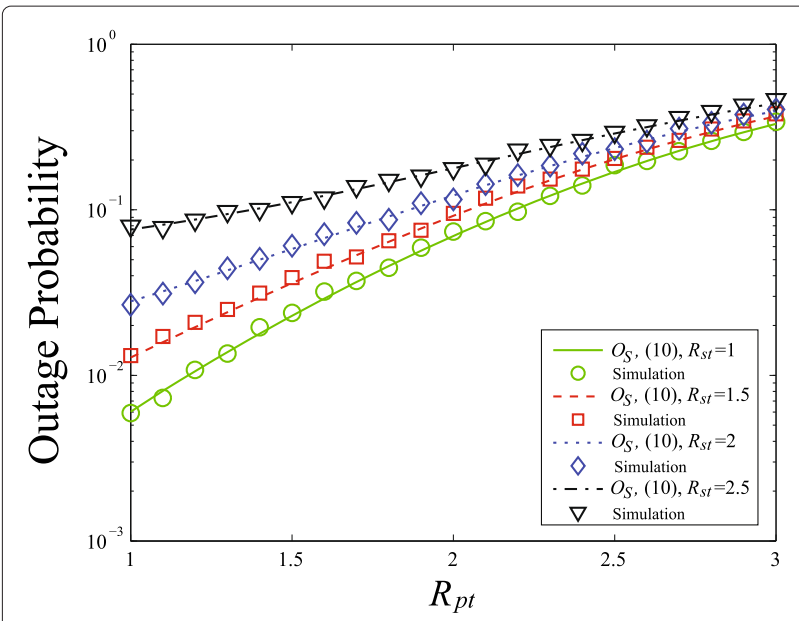

Fig. 11 The end-to-end outage probability $O_{S}$ with respect to $R_{p t}$ where $\alpha=\alpha^{*}$ and $P_{S}=10 \mathrm{~dB}$

For a better illustration of Theorem 2, $O_{P}$ is plotted with respect to $R_{p t}$ in Fig. 8 . With an increase in $R_{p t}$, the corresponding outage performance of the primary system is degraded. Whereas with an increase in $\alpha$, a better outage performance is achieved. When $\alpha=\alpha^{*}=\frac{R_{p t}^{\prime}}{R_{p t}^{\prime}+1}$, the corresponding performance outperforms that with fixed power allocation factor, e.g., $\alpha=0.8,0.9$, thus validating Theorem 2 that the end-to-end outage performance of the primary system is optimized with respect to $\alpha$ when $\alpha \geq \alpha^{*}$. Similar results can be observed for the benchmark case [28]. Again, a better performance is achieved by the proposed approach compared to that in [28]. Furthermore, in modest rate region where $R_{p t}<2.2$, the condition in (32) is satisfied and a performance gain is achieved for the primary system compared to the benchmark case. Whereas in the high rate region, since both the SIC decoding at CR and the decoding at PR become more difficult, the primary user experiences a performance loss.

Figure 9 displays $O_{P}$ with respect to $P_{P}$ where a power allocation factor of $\alpha=\alpha^{*}$ is adopted at CR. When $P_{S}=$ $10 \mathrm{~dB}$, it is observed that a diversity order of 2 is achieved for the primary system when $P_{P} \rightarrow \infty$. This is reasonable as in the proposed protocol, two independent copies of $x_{p}$ are received at $\mathrm{PR}$ from $\mathrm{PT} \rightarrow \mathrm{PR}$ and $\mathrm{CR} \rightarrow \mathrm{PR}$ respectively in two successive phases. However, when there is a fixed power ratio between $P_{P}$ and $P_{S}$, i.e., $P_{S}=\theta P_{P}$, it is observed that the performance of the primary system is limited by the interference from secondary transmissions and no diversity gain is achieved.

5.3 End-to-end outage performance of the secondary user In Fig. 10, the end-to-end outage probability of the secondary user $O_{S}$ is plotted with respect to $\alpha$ where $P_{P}=30$ $\mathrm{dB}$ and $P_{S}=\theta P_{P}$. It is observed that $O_{S}$ experiences a plateau when $\alpha$ takes the values between 0.3 and 0.7. And when $\alpha$ is less than 0.25 or greater than 0.75 , a reasonably good outage performance is achieved for the secondary user. This is because of the employment of SIC at SR. In the regions where $\alpha \leq 0.25$ and $\alpha \geq 0.75$, there is a significant difference between the power levels of $x_{p}$ and $x_{s}$ received at SR in the second phase, thus $x_{s}$ can either be first decoded or successively decoded using SIC with a high probability. Conversely, when $\alpha$ takes values between 0.3 and 0.7 , SIC is limited by the comparable interference between $x_{p}$ and $x_{s}$, which makes it difficult to decode either of them. For comparison purposes, the outage performance of the secondary user in the benchmark case [28] is also presented, where SR attempts to recover the desired signal $x_{s}$ by using MRC of the received signals in two phases and considers the primary component simply as noise. As illustrated in Fig. 10, with an increase in $\alpha$, since more power is allocated to forward the primary signal meanwhile higher interference is seen at SR, the outage performance of the secondary user is severely degraded.

Furthermore, it is observed from Fig. 10 that the performance of the secondary system is degraded with an increase in $\theta$. This is because with a higher $\theta$, e.g., $\theta$ is increased from -20 to $-15 \mathrm{~dB}$, from (34a), it becomes difficult to first decode and remove $x_{p}$ and then decode $x_{s}$ successively using SIC. In other words, it is not always beneficial to adopt a high transmit power at ST, even if the condition in (32) is met. Again, the outage performance using DPC at CR is illustrated in Fig. 10. Conditioned on event $\mathcal{E}^{(1)}$, by using DPC to pre-cancel the interference seen at SR, it is observed that a performance upper bound is achieved for the secondary user.

Together with Figs. 7 and 10, when $\alpha \geq \alpha^{*}$, it is possible to bring a performance gain for the primary system and at the same time achieve a reasonably good outage performance around $10^{-2}$ for the secondary system by the proposed approach. Whereas for the benchmark case considered in [28], although it is also possible to provide a performance gain to the primary user when $\alpha \geq \alpha^{*}$, the $\mathrm{s}$ econdary message $x_{s}$ can be hardly delivered from ST to SR.

In Fig. 11, $O_{S}$ is plotted with respect to $R_{p t}$ when $\alpha=\alpha^{*}$. Similarly, with an increase in $R_{p t}$, from (34a), it becomes difficult to decode $x_{p}$ first and then decode $x_{s}$ successively using SIC, thus the corresponding outage performance of the secondary system is degraded. On the other hand, with other parameters being the same, it is observed that a performance degradation is experienced by the secondary system with an increase in $R_{s t}$.

From the above observations in Figs. 4, 5, 6, 7, 8, 9, 10, and 11 , with properly designed parameters to facilitate the SIC decoding at CR as well as to limit the interference caused to PR due to the cross talk in the first phase, it is possible to find a suitable power allocation factor $\alpha \geq \alpha^{*}$ such that a performance gain is achieved for the primary 
user meanwhile the secondary user gains an opportunity to access the spectrum. Furthermore, with the same system parameters, performance gains are achieved for both primary and secondary systems by the proposed approach compared to a benchmark case in [28].

\section{Conclusions}

In this paper, the interference channel with a cognitive relay is exploited to achieve spectrum sharing between a licensed primary user and an unlicensed secondary user. A causal cognitive two-phase spectrum sharing protocol is proposed and closed-form expressions of the end-toend outage probability are derived. In view of the inherent interference-limited property of the system, to guarantee the performance of the primary system, we consider a power control at ST together with a power allocation at CR to forward the processed primary and secondary messages, respectively. Simulation results demonstrate that by designing both the power control at ST and power allocation at $C R$, spectrum sharing is achieved between the primary and secondary systems and performance gains can be achieved for both parties.

\section{Appendix A: Derivations of $\operatorname{Pr}\left\{\mathcal{E}^{(1)}\right\}, \operatorname{Pr}\left\{\mathcal{E}^{(2)}\right\}$, $\operatorname{Pr}\left\{\mathcal{E}^{(3)}\right\}$, and $\operatorname{Pr}\left\{\mathcal{E}^{(4)}\right\}$}

From Fig. 3, upon determining the region of event $\mathcal{E}^{(1)}$ that is defined in (19a), the corresponding probability can be derived by

$$
\begin{aligned}
\operatorname{Pr}\left\{\mathcal{E}^{(1)}\right\}= & \operatorname{Pr}\left\{\left(\mathcal{E}_{1, p} \cap \mathcal{E}_{2, s}\right) \cup\left(\mathcal{E}_{1, s} \cap \mathcal{E}_{2, p}\right)\right\} \\
= & \int_{\frac{R_{s t}^{\prime}}{P_{S}}}^{\infty} \delta_{s r} e^{-\delta_{s r} \gamma_{s r}}\left[\int_{\frac{R_{p t}^{\prime} P_{S}}{P_{P}} \gamma_{s r}+\frac{R_{p t}^{\prime}}{P_{P}}}^{\infty} \delta_{p r} e^{-\delta_{p r} \gamma_{p r}} d \gamma_{p r}\right] d \gamma_{s r} \\
& +\int_{\frac{R_{p t}^{\prime}}{P_{P}}}^{\infty} \delta_{p r} e^{-\delta_{p r} \gamma_{p r}}\left[\int_{\frac{R_{s t}^{\prime} P_{P}}{P_{S}} \gamma_{p r}+\frac{R_{s t}^{\prime}}{P_{S}}}^{\infty} \delta_{s r} e^{-\delta_{s r} \gamma_{s r}} d \gamma_{s r}\right] d \gamma_{p r},
\end{aligned}
$$

where $\delta_{s r} e^{-\delta_{s r} \gamma_{s r}}$ and $\delta_{p r} e^{-\delta_{p r} \gamma_{p r}}$ denote the respective PDFs of $\gamma_{s r}$ and $\gamma_{p r}$.

For event $\mathcal{E}^{(2)}$ defined in (19b), the corresponding probability can be derived by

$$
\begin{aligned}
\operatorname{Pr}\left\{\mathcal{E}^{(2)}\right\} & =\operatorname{Pr}\left\{\mathcal{E}_{1, p} \cap \overline{\mathcal{E}}_{2, s}\right\} \\
& =\int_{0}^{\frac{R_{s t}^{\prime}}{P_{S}}} \delta_{s r} e^{-\delta_{s r} \gamma_{s r}}\left[\int_{\frac{R_{p t}^{\prime} P_{S}}{P_{P}} \gamma_{s r}+\frac{R_{p t}^{\prime}}{P_{P}}}^{\infty} \delta_{p r} e^{-\delta_{p r} \gamma_{p r}} d \gamma_{p r}\right] d \gamma_{s r} .
\end{aligned}
$$

For event $\mathcal{E}^{(3)}$ defined in (19c), the corresponding probability can be derived by

$$
\begin{aligned}
\operatorname{Pr}\left\{\mathcal{E}^{(3)}\right\} & =\operatorname{Pr}\left\{\mathcal{E}_{1, s} \cap \overline{\mathcal{E}}_{2, p}\right\} \\
& =\int_{0}^{\frac{R_{p t}^{\prime}}{P_{P}}} \delta_{p r} e^{-\delta_{p r} \gamma_{p r}}\left[\int_{\frac{R_{s t}^{\prime} P_{P}}{P_{S}} \gamma_{p r}+\frac{R_{s t}^{\prime}}{P_{S}}}^{\infty} \delta_{s r} e^{-\delta_{s r} \gamma_{s r}} d \gamma_{s r}\right] d \gamma_{p r}
\end{aligned}
$$

Then the integrations in (53)-(55) can be solved to obtain the results in (21a)-(21c). Since events $\mathcal{E}^{(1)}, \mathcal{E}^{(2)}$, $\mathcal{E}^{(3)}$, and $\mathcal{E}^{(4)}$ are mutually exclusive, the corresponding probability of event $\mathcal{E}^{(4)}$ can be readily obtained by

$$
\operatorname{Pr}\left\{\mathcal{E}^{(4)}\right\}=1-\operatorname{Pr}\left\{\mathcal{E}^{(1)}\right\}-\operatorname{Pr}\left\{\mathcal{E}^{(2)}\right\}-\operatorname{Pr}\left\{\mathcal{E}^{(3)}\right\} .
$$

\section{Appendix B: Derivations of $O_{P}^{(1)}$}

From (22), with target rate $R_{p t}$, we have the corresponding outage probability at PR

$$
\begin{aligned}
O_{P}^{(1)} & =\operatorname{Pr}\left\{R_{p}^{(1)}<R_{p t}\right\} \\
& \approx \operatorname{Pr}\left\{C\left(\frac{P_{P} \gamma_{p p}}{P_{S} \gamma_{s p}+1}+\frac{\alpha}{1-\alpha}\right)<R_{p t}\right\} \\
& =\left\{\begin{array}{ll}
0, & \alpha \geq \frac{R_{p t}^{\prime}}{R_{p t}^{\prime}+1} \\
\operatorname{Pr}\left\{\gamma_{p p}<\frac{\left(R_{p t}^{\prime}-\frac{\alpha}{1-\alpha}\right) P_{S}}{P_{P}} \gamma_{s p}+\frac{R_{p t}^{\prime}-\frac{\alpha}{1-\alpha}}{P_{P}}\right\}, \alpha<\frac{R_{p t}^{\prime}}{R_{p t}^{\prime}+1}
\end{array} .\right.
\end{aligned}
$$

When $\alpha<\frac{R_{p t}^{\prime}}{R_{p t}^{\prime}+1}$, similarly, we can derive $O_{P}^{(1)}$ by integrating over the corresponding region

$$
\begin{aligned}
& \operatorname{Pr}\left\{\gamma_{p p}<\frac{\left(R_{p t}^{\prime}-\frac{\alpha}{1-\alpha}\right) P_{S}}{P_{P}} \gamma_{s p}+\frac{R_{p t}^{\prime}-\frac{\alpha}{1-\alpha}}{P_{P}}\right\} \\
& =\int_{0}^{\infty} \delta_{s p} e^{-\delta_{s p} \gamma_{s p}}\left[\int_{0}^{\left.\frac{\left(R_{p t}^{\prime}-\frac{\alpha}{1-\alpha}\right) P_{S}}{P_{P}} \gamma_{s p}+\frac{R_{p t}^{\prime}-\frac{\alpha}{1-\alpha}}{P_{P}} \delta_{p p} e^{-\delta_{p p} \gamma_{p p}} d \gamma_{p p}\right] d \gamma_{s p},}\right.
\end{aligned}
$$

where $\delta_{s p} e^{-\delta_{s p} \gamma_{s p}}$ and $\delta_{p p} e^{-\delta_{p p} \gamma_{p p}}$ denote the respective PDFs of $\gamma_{s p}$ and $\gamma_{p p}$. Then, this integration can be solved to obtain the results in (23).

\section{Appendix C: Derivations of $\boldsymbol{O}_{P}^{(4)}$}

From (28), with target rate $R_{p t}$, we have the corresponding outage probability at PR

$$
\begin{aligned}
O_{P}^{(4)} & =\operatorname{Pr}\left\{R_{p}^{(4)}<R_{p t}\right\} \\
& =\operatorname{Pr}\left\{\gamma_{p p}<\frac{R_{p t}^{\prime} P_{S}}{2 P_{P}} \gamma_{s p}+\frac{R_{p t}^{\prime}}{2 P_{P}}\right\} .
\end{aligned}
$$

By integrating over the corresponding region defined in (59), $O_{P}^{(4)}$ can be derived by

$$
O_{P}^{(4)}=\int_{0}^{\infty} \delta_{s p} e^{-\delta_{s p} \gamma_{s p}}\left[\int_{0}^{\frac{R_{p t}^{\prime} P_{S}}{2 P_{P}} \gamma_{s p}+\frac{R_{p t}^{\prime}}{2 P_{P}}} \delta_{p p} e^{-\delta_{p p} \gamma_{p p}} d \gamma_{p p}\right] d \gamma_{s p}
$$

Then, this integration can be solved to obtain the result in (29). 


\section{Appendix D: Derivations of $O_{S}^{(1)}$}

From (35), the outage probability at SR can be expressed as [34]

$$
\begin{aligned}
O_{S}^{(1)}= & 1-\operatorname{Pr}\left\{\mathcal{E}_{1, s}^{(1)} \cup\left(\mathcal{E}_{1, p}^{(1)} \cap \mathcal{E}_{2, s}^{(1)}\right)\right\} \\
= & 1-\operatorname{Pr}\left\{\mathcal{E}_{1, s}^{(1)}\right\}-\operatorname{Pr}\left\{\mathcal{E}_{1, p}^{(1)} \cap \mathcal{E}_{2, s}^{(1)}\right\} \\
& +\operatorname{Pr}\left\{\mathcal{E}_{1, s}^{(1)} \cap \mathcal{E}_{1, p}^{(1)} \cap \mathcal{E}_{2, s}^{(1)}\right\} .
\end{aligned}
$$

Since $R_{1, s}^{(1)} \leq R_{2, s}^{(1)}$ always holds, we have $\mathcal{E}_{1, s}^{(1)} \subseteq \mathcal{E}_{2, s}^{(1)}$, thus (61) can be rewritten as

$$
O_{S}^{(1)}=1-\operatorname{Pr}\left\{\mathcal{E}_{1, s}^{(1)}\right\}-\operatorname{Pr}\left\{\mathcal{E}_{1, p}^{(1)} \cap \mathcal{E}_{2, s}^{(1)}\right\}+\operatorname{Pr}\left\{\mathcal{E}_{1, p}^{(1)} \cap \mathcal{E}_{1, s}^{(1)}\right\} .
$$

From (34), by integrating over the corresponding regions of the events in (62), we have

$$
\begin{aligned}
& \operatorname{Pr}\left\{\mathcal{E}_{1, s}^{(1)}\right\} \approx \int_{\frac{R_{s t}^{\prime} \frac{1-\alpha}{\alpha}}{P_{S}}}^{\infty} \delta_{s s} e^{-\delta_{s s} \gamma_{s s}}\left[\int_{0}^{\frac{P_{S}}{\left(R_{s t}^{\prime}-\frac{1-\alpha}{\alpha}\right) P_{P}} \gamma_{s s}-\frac{1}{P_{P}}} \delta_{p s} e^{-\delta_{p s} \gamma_{p s}} d \gamma_{p s}\right] d \gamma_{s s}, \\
& \operatorname{Pr}\left\{\mathcal{E}_{1, p}^{(1)} \cap \mathcal{E}_{2, s}^{(1)}\right\} \approx
\end{aligned}
$$

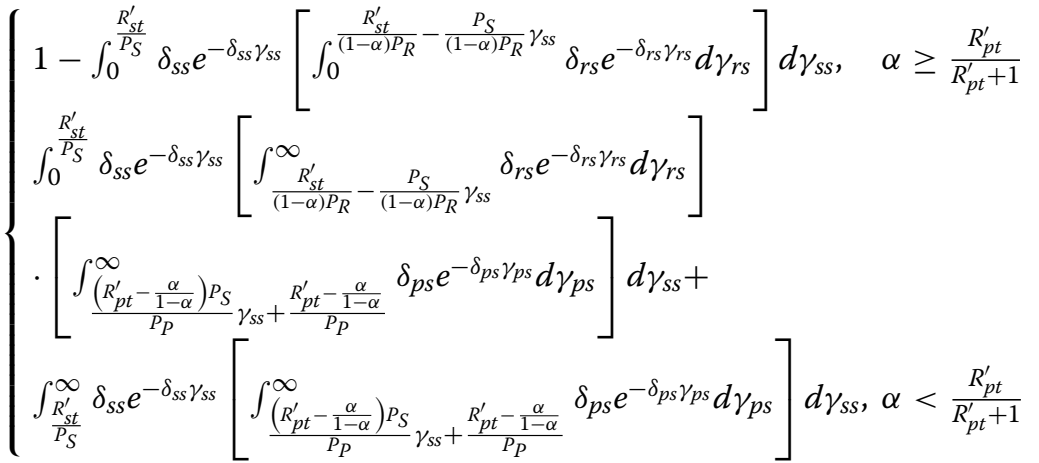

$$
\begin{aligned}
& \operatorname{Pr}\left\{\mathcal{E}_{1, p}^{(1)} \cap \mathcal{E}_{1, s}^{(1)}\right\} \approx
\end{aligned}
$$

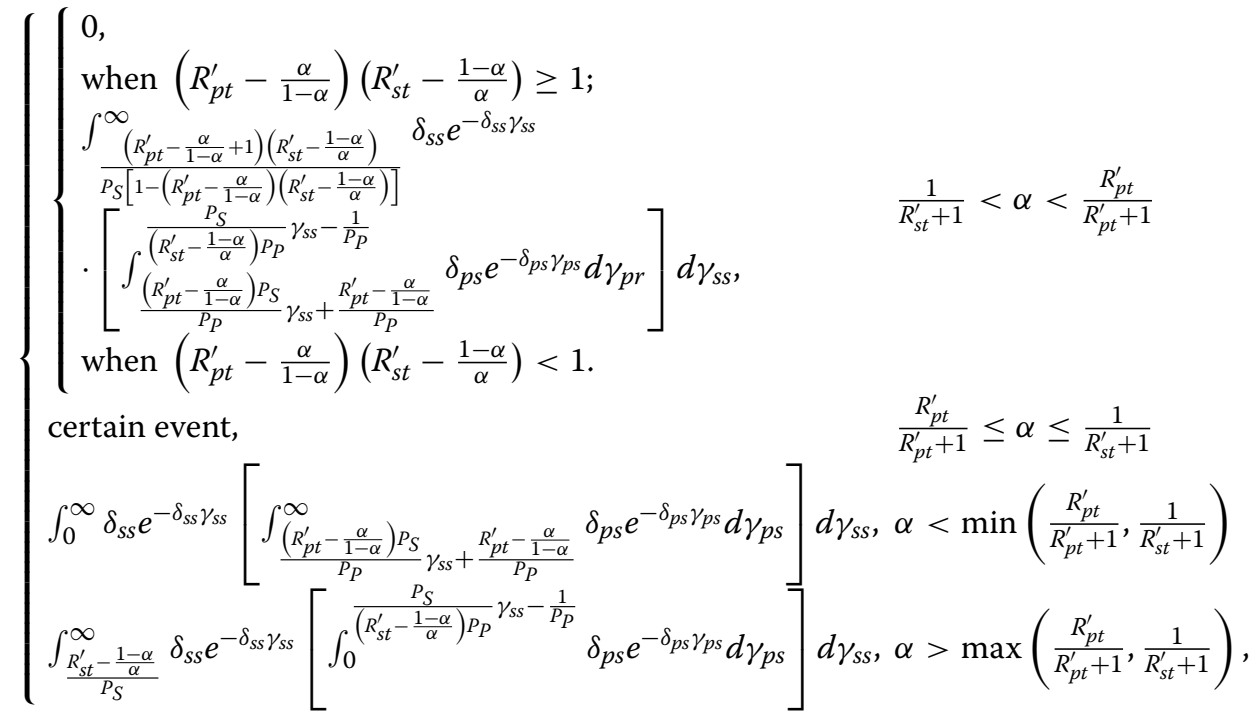

respectively. Then, these integrations can be solved to obtain the results in (37a)-(37c). 


\section{Competing interests}

The authors declare that they have no competing interests.

\section{Acknowledgements}

The authors would like to acknowledge the support from the Ministry of Science and Technology (MOST) of China under the grants 2014DFA11640 and 2015DFG12580, the National Natural Science Foundation of China (NSFC) under the grants 61301128,61461136004, and 61271224, the NFSC Major International Joint Research Project under the grant 61210002, the Hubei Provincial Science and Technology Department under the grants 2011 BFA004 and 2013BHE005, the Fundamental Research Funds for the Central Universities under the grant 2015XJGH011, and the Special Research Fund for the Doctoral Program of Higher Education (SRFDP) under the grant 20130142120044. This research is partially supported by EU FP7-PEOPLE-IRSES, project acronym S2EuNet (grant no. 247083), project acronym WiNDOW (grant no. 318992), and project acronym CROWN (grant no. 610524).

\section{Author details}

${ }^{1}$ School of Electronic Information and Communications, Huazhong University of Science and Technology, Wuhan, China. ${ }^{2}$ Philips Research, High Tech Campus, 5656 AE Eindhoven, Netherlands. ${ }^{3}$ Ruckus Wireless, Singapore, Singapore.

\section{Received: 29 September 2014 Accepted: 3 June 2015}

Published online: 26 June 2015

\section{References}

1. H Cheng, Y-D Yao, Cognitive-relay-based intercell interference cancellation in cellular systems. IEEE Trans. Veh. Tech. 59(4), 1901-1909 (2010)

2. D Li, Cognitive relay networks: opportunistic or uncoded decode-and-forward relaying. IEEE Trans. Veh. Tech. 63(3), 1486-1491 (2014)

3. JN Laneman, DNC Tse, GW Wornell, Cooperative diversity in wireless networks: efficient protocols and outage behavior. IEEE Trans. Inf. Theory. 50(12), 3062-3080 (2004)

4. G Kramer, M Gastpar, P Gupta, Cooperative strategies capacity theorems for relay networks. IEEE Trans. Inf. Theory. 51(9), 3037-3063 (2005)

5. X Zhang, W Wang, X Ji, Multiuser diversity in multiuser two-hop cooperative relay wireless networks: system model and performance analysis. IEEE Trans. Veh. Tech. 58(2), 1031-1036 (2009)

6. F Yang, M Huang, M Zhao, S Zhang, W Zhou, Cooperative strategies for wireless relay networks with cochannel interference over time-correlated fading channels. IEEE Trans. Veh. Tech. 62(7), 3392-3408 (2013)

7. O Sahin, E Erkip, in Proceedings of IEEE Global Telecommunications Conference (GLOBECOM). Achievable rates for the gaussian interference relay channel (IEEE Washington, DC, 2007), pp. 1627-1631

8. O Sahin, E Erkip, in Proceedings of 41 st Asilomar Conference on Signals, Systems and Computers (ACSSC). On achievable rates for interference relay channel with interference cancelation (IEEE Pacific Grove, CA, 2007), pp. 805-809

9. S Sridharan, S Vishwanath, S Jafar, S Shamai, in Proceedings of IEEE International Symposium on Information Theory (ISIT). On the capacity of cognitive relay assisted Gaussian interference channel (IEEE Toronto, ON, 2008), pp. 549-553

10. SRini, D Tuninetti, N Devroye, in Proceedings of IEEE Information Theory Workshop (ITW). Outer bounds for the interference channel with a cognitive relay (IEEE Dublin, Ireland, 2010), pp. 1-5

11. Y Tian, A Yener, The Gaussian interference relay channel: improved achievable rates and sum rate upperbounds using a potent relay. IEEE Trans. Inf. Theory. 57(5), 2865-2879 (2011)

12. S Rini, D Tuninetti, N Devroye, New inner and outer bounds for the memoryless cognitive interference channel and some new capacity results. IEEE Trans. Inf. Theory. 57(7), 4087-4109 (2011)

13. S Rini, D Tuninetti, N Devroye, Inner and outer bounds for the Gaussian cognitive interference channel and new capacity results. IEEE Trans. Inf. Theory. 58(2), 820-848 (2012)

14. H Charmchi, GA Hodtani, M Nasiri-Kenari, A new outer bound for a class of interference channels with a cognitive relay and a certain capacity result. IEEE Commun. Lett. 17(2), 241-244 (2013)

15. S Rini, D Tuninetti, N Devroye, AJ Goldsmith, On the capacity of the interference channel with a cognitive relay. IEEE Trans. Inf. Theory. 60(4), 2148-2179 (2014)
16. TS Han, K Kobayashi, A new achievable rate region for the interference channel. IEEE Trans. Inf. Theory. 27(1), 49-60 (1981)

17. M Costa, Writing on dirty paper. IEEE Trans. Inf. Theory. 29(3), 439-441 (1983)

18. O Sahin, O Simeone, E Erkip, Interference channel with an out-of-band relay. IEEE Trans. Inf. Theory. 57(5), 2746-2764 (2011)

19. Y Tian, A Yener, Symmetric capacity of the gaussian interference channel with an out-of-band relay to within 1.15 bits. IEEE Trans. Inf. Theory. 58(8), 5151-5171 (2012)

20. S Haykin, Cognitive radio: brain-empowered wireless communications. IEEE J. Select Areas Commun. 23(2), 201-220 (2005)

21. Y Xing, CN Mathur, MA Haleem, R Chandramouli, KP Subbalakshmi, Dynamic spectrum access with QoS and interference temperature constraints. IEEE Trans. Mobile Comput. 6(4), 423-433 (2007)

22. A Goldsmith, SA Jafar, I Marić, S Srinivasa, Breaking spectrum gridlock with cognitive radios: an information theoretic perspective. Proc. IEEE. 97(5), 894-914 (2009)

23. Y-C Liang, K-C Chen, GY Li, P Mahonen, Cognitive radio networking and communications: an overview. IEEE Trans. Veh. Tech. 60(7), 3386-3407 (2011)

24. Y Han, A Pandharipande, TS Ting, Cooperative decode-and-forward relaying for secondary spectrum access. IEEE Trans. Wireless Commun. 8(10), 4945-4950 (2009)

25. Q Li, SH Ting, A Pandharipande, Y Han, Cognitive spectrum sharing with two-way relaying systems. IEEE Trans. Veh. Tech. 60(3), 1233-1240 (2011)

26. A Jovičić, P Viswanath, Cognitive radio: an information-theoretic perspective. IEEE Trans. Inf. Theory. 55(9), 3945-3958 (2009)

27. $\mathrm{M}$ Cheng, J Mao, L Li, in Proceedings of 4 th International Conference on Wireless Communications Networking and Mobile Computing (WiCOM). Investigation of mutual interference channel with relay in cognitive transmission (IEEE Dalian, China, 2008), pp. 1-4

28. W Jaafar, W Ajib, D Haccoun, in Proceedings of IEEE Global Communications Conference (GLOBECOM). A novel relay-aided transmission scheme in cognitive radio networks (IEEE Houston, TX, 2011), pp. 1-6

29. W Jaafar, W Ajib, D Haccoun, in Proceedings of IEEE International Conference on Communications (ICC). Opportunistic adaptive relaying in cognitive radio networks (IEEE Kyoto, Japan, 2012), pp. 1811-1815

30. TMC Chu, H Phan, $\mathrm{H}-J$ Zepernick, in Proceedings of 23rd IEEE International Symposium on Personal, Indoor and Mobile Radio Communications (PIMRC). Amplify-and-forward relay assisting both primary and secondary transmissions in cognitive radio networks over nakagami-m fading (IEEE Sydney, Australia, 2012), pp. 932-937

31. Q Li, A Pandharipande, SH Ting, in Proceedings of IEEE Wireless Communications and Networking Conference (WCNC). Spectrum sharing on interference channels with a cognitive relay (IEEE Shanghai, China, 2013) pp. 2982-2987

32. TM Cover, JA Thomas, Elements of Information Theory, 2nd edition. (John Wiley \& Sons, Inc., Hoboken, New Jersey, 2006)

33. Q Li, SH Ting, A Pandharipande, Y Han, Adaptive two-way relaying and outage analysis. IEEE Trans. Wireless Commun. 8(6), 3288-3299 (2009)

34. A Papoulis, SU Pillai, Probability, Random Variables and Stochastic Processes, 4th Edition. (McGraw Hill, New York, 2002)

35. V Asghari, S Aissa, End-to-end performance of cooperative relaying in spectrum-sharing systems with quality of service requirements. IEEE Trans. Veh. Tech. 60(6), 2656-2668 (2011)

36. K Tourki, KA Qaraqe, M-S Alouini, Outage analysis for underlay cognitive networks using incremental regenerative relaying. IEEE Trans. Veh. Tech. $\mathbf{6 0}(2), 721-734(2013)$

37. F Guimaraes, D Da Costa, T Tsiftsis, C Cavalcante, G Karagiannidis, Multiuser and multirelay cognitive radio networks under spectrum-sharing constraints. IEEE Trans. Veh. Tech. 63(1), 433-439 (2014) 